\title{
The Cream of the Crop: Biology, Breeding and Applications of Cannabis sativa
}

\author{
Susanne Schilling $*^{1}$, CarolineA.Dowling $*^{1}$, JiaqiShi $*$ \\ ${ }^{1}$, LouiseRyan $^{1}$, DavidHunt $^{1}$, EveOReilly ${ }^{1}$, AntoinetteS.Perry ${ }^{1}$, Oliver Kinnane $^{1}$, PaulF.McCabe $^{1}$, andl \\ ${ }^{1}$ University College Dublin
}

October 1, 2020

\begin{abstract}
Cannabis sativa is an extraordinarily versatile species. Hemp and its cousin marijuana, both C. sativa, have been used for millennia as a source of fibre, oil and for medicinal, spiritual and recreational purposes. Because the consumption of Cannabis can have psychoactive effects, the plant has been widely banned throughout the last century. In the past decade, evidence of its medicinal properties did lead to the relaxation of legislation in many countries around the world. Consequently, the genetics and development of Cannabis as well as Cannabis-derived products are the subject of renewed attention. Here, we review the biology of C. sativa, including recent insights from taxonomy, morphology and genomics, with an emphasis on the genetics of cannabinoid synthesis. Because the female Cannabis flower is of special interest as the site of cannabinoid synthesis, we explore flower development, flowering time well as the species' unique sex determination system in detail. Furthermore, we outline the tremendous medicinal, engineering, and environmental opportunities that Cannabis bears. Together, the picture emerges that our understanding of Cannabis biology currently progresses at an unusual speed. A future challenge will be to preserve the multi-purpose nature of Cannabis, and to harness its medicinal properties and sustainability advantages simultaneously.

*: These authors contributed equally to this work and should be considered joint first authors

^: for correspondence: susanne.schilling [at] udc.ie; rainer.melzer[at]ucd.ie

Keywords: building materials, Cannabis sativa, cannabinoids, crop breeding, flower development, flowering time, hemp, marijuana, sex determination, sustainability

\section{Introduction - Cannabis in a nutshell}

Cannabis sativa is a highly versatile crop with dozens of different uses (Figure 1). There are a multitude of medical applications for Cannabis secondary compounds, which have been shown to reduce pain, nausea and neurological conditions like seizures (Whiting et al., 2015), and research on effects on inflammation, depression and cancer is also being conducted (Atalay et al., 2019; Fraguas-Sánchez and Torres-Suárez, 2018; Poleszak et al., 2018; Śledziński et al., 2018, Russo, 2011). Beyond that, fibre varieties of Cannabis have high carbon sequestering potential because of their rapid growth. They are therefore utilized for carbon storage in building materials or as biofuel (Finnan and Styles, 2013). For those different reasons, the Cannabis industry is gaining more traction and the need for specialized varieties, adapted to local climatic conditions, or suited for specific applications, is steadily increasing.
\end{abstract}




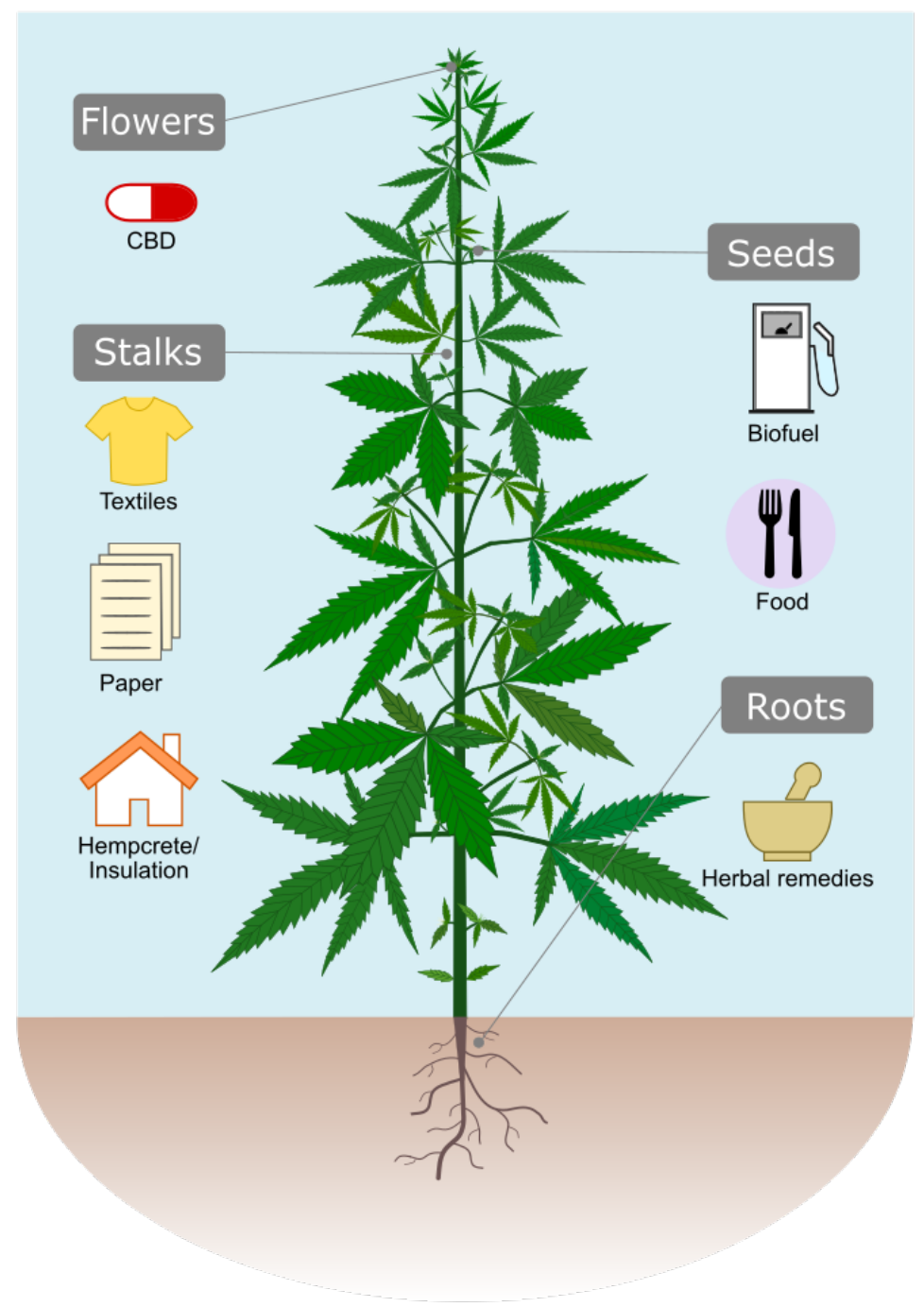

Figure 1: Cannabis is a multipurpose crop. The uses for Cannabis are manifold. The trichomes present on female flowers produce efficacious phytocannabinoids including cannabidiol (CBD), that has a wide range of medical uses that have been demonstrated in clinical trials. The stalks of the Cannabis plant can be processed into textiles, paper and building materials. The roots of the hemp plant have been used in traditional herbal remedies. The seeds can be processed for biofuel or pressed for oil for human consumption. Furthermore, the seed cake remaining after oil is pressed is protein-rich and can be used as animal feed. See also Figure 9 for photos of hemp products.

Cannabis is probably best known for one secondary compound, the psychotropic substance tetrahydrocannabinol (THC). Depending on the THC content of the plant or more specifically the dried inflorescence, Cannabis is either classified as marijuana (or drug-type, plants above $0.3 \% \mathrm{THC}$ ) or hemp (fibre-type, below 
$0.3 \%$ THC), which is mainly a legal and not a strict taxonomic classification. A more refined classification of Cannabis according to the phytocannabinoid profile into distinct 'chemotypes' can also be useful, with chemotype I and II being marijuana while chemotypes III, IV and V can be seen as hemp (see chapter 3).

Many countries have been easing the ban on medical and even recreational use of THC during the past decade. However, because of the prohibition of Cannabis in many countries throughout the last century, it was not bred to the same extent as other high-value crops. Hence, hemp and marijuana lines retain a high level of genetic variability and heterozygosity, that is not found in other crops (Sawler et al., 2015).

Here, we review the biology as well as the applications and future perspectives of Cannabis research and breeding. We discuss Cannabis taxonomy and cannabinoid synthesis as well as flower development and flowering time control with an emphasis on sex determination in this predominantly dioecious species. We also summarize the currently available genomics resources. Since Cannabis is so versatile, we discuss its applications in medicine as well as in the building industry. Cannabis ' future role in a sustainable society is summarized as well as the future of cannabinoid production via cell suspension cultures.

\section{Cannabis systematics}

Cannabis is the botanical name of a genus that historically includes three species, C. sativa, C. ruderalis and $C$. indica. However, since the three species can intercross, they are also often considered one single species, C. sativa (Small, 2015). Recent genetic data support the single-species concept and recommend that three subspecies should be recognized: Cannabis sativa subsp. sativa, subsp. indica and subsp. ruderalis (Q. Zhang et al., 2018).

Cannabis is a dioecious species, meaning there are male and female individuals (Figure 2a-c). However, through breeding, monoecious lines with male and female flowers on the same plant have also been generated (Figure 2d) (Moliterni et al., 2004).

The genus Cannabis is part of the Cannabaceae, a small family of flowering plants with 10 genera and some 120 species (Jin et al., 2020; Yang et al., 2013). The Cannabaceae have been estimated to have originated ca. 70 to 90 million years ago, and are distributed in temperate and tropical regions throughout the world (Figure 3) (Jin et al., 2020; Magallón et al., 2015). Most species of the Cannabaceae are trees or shrubs, Cannabis as a herb is, therefore, the exception rather than the rule in the family. However, a trait Cannabis shares with many other species in the family is the inconspicuous unisexual flowers (Yang et al., 2013).

The closest relative of Cannabis is the genus Humulus(Yang et al., 2013), which consists of three species, among which Humulus lupulus(hop) is economically important for the beer brewing industry. Both hop and Cannabis produce separate male and female flowers, and the trichomes in the female inflorescences are the site of secondary compound production that make both of those plants economically valuable (Page and Nagel, 2006).

Within the angiosperm phylogeny, Cannabaceae are most closely related to the Moraceae (mulberry or fig family) and Urticaceae (nettle family). Together with the Ulmaceae (elms and relatives) they form a group known as the urticalean rosids (Figure 3) (Sytsma et al., 2002). It is interesting to note that unisexual flowers appear to be prevalent in the urticalean rosids, whereas bisexual flowers are by far the dominant system in angiosperms in general (Renner, 2014; Sytsma et al., 2002). The evolution of sex expression and sex determination in this group is an interesting area of future research.

The urticalean rosids belong to the order Rosales, which are eudicots (The Angiosperm Phylogeny Group, 2016). Though the Rosales comprise some 7700 species (Zhang et al., 2011), they contain relatively few wellcharacterized model plants. The flowering plant super-models Arabidopsis thaliana (thale cress, Brassicales) and Oryza sativa (rice, monocots) are only distantly related to Cannabis, the lineages leading to Arabidopsis 

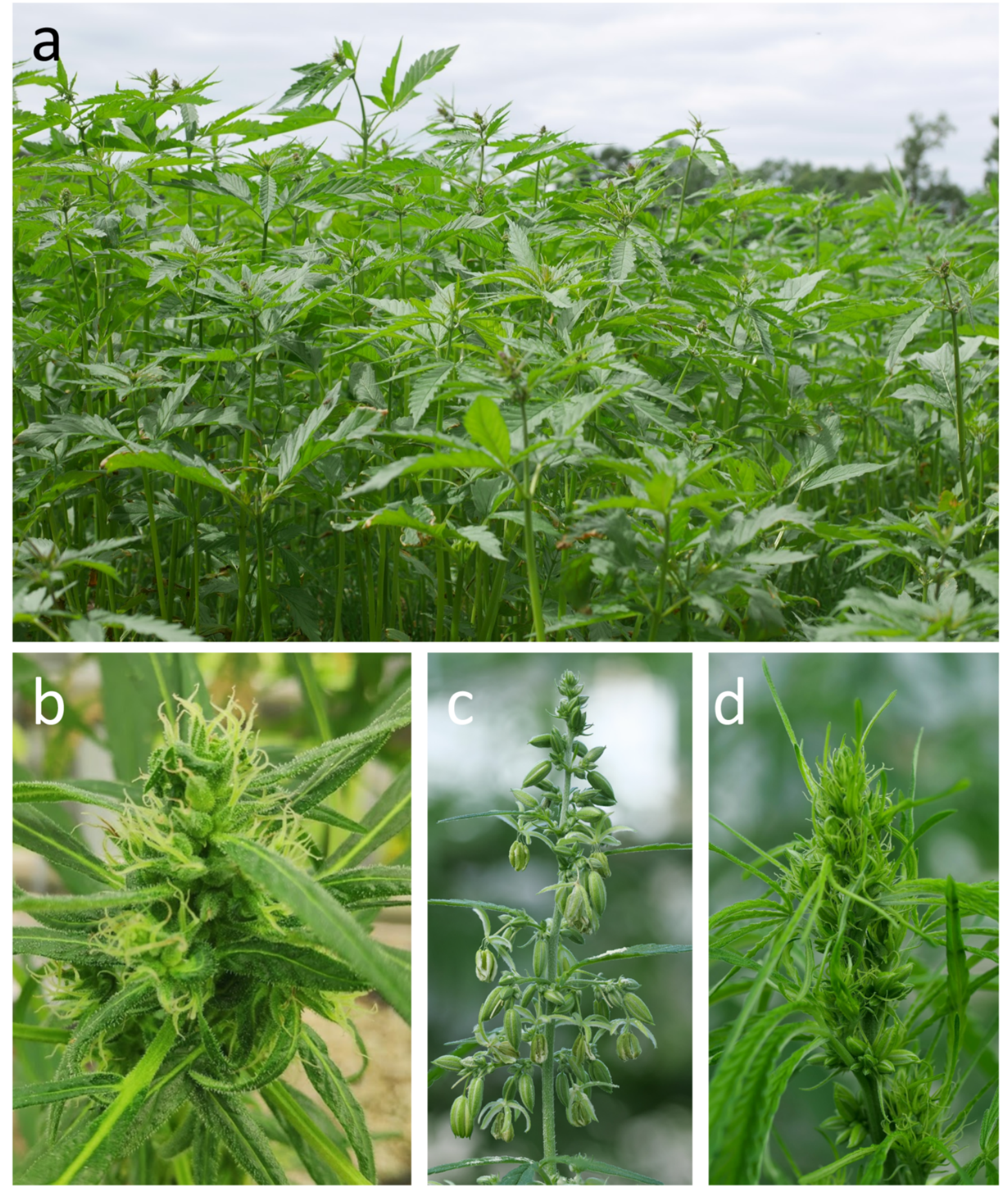

Figure 2: Hemp varieties of Cannabis sativa. Cannabis plants of the hemp cultivar 'Finola' growing in the field (a). The cultivar is dioecious with female (b) and male individuals (c). Monoecious plants of the cultivar 'Felina 32' show male flowers and female flowers in one individual plant (d). 


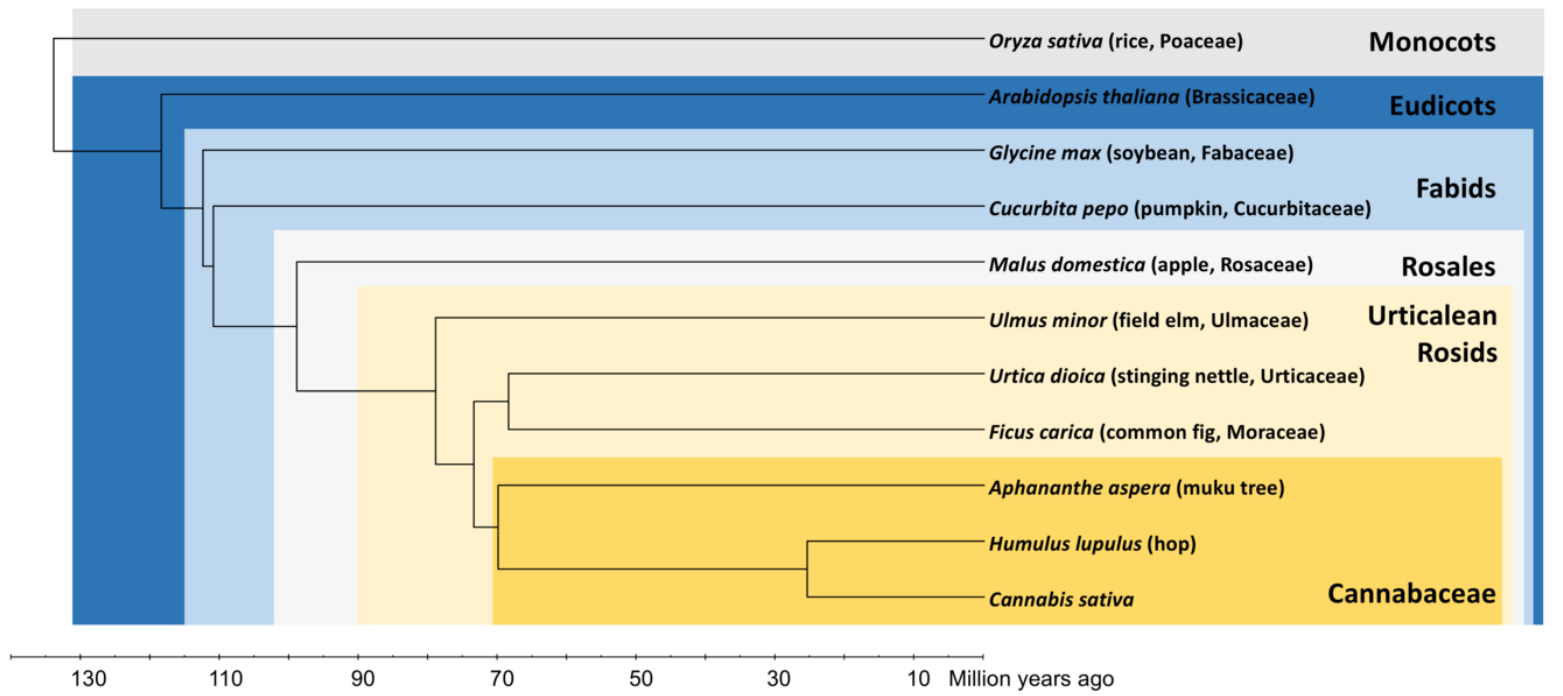

Figure 3: Phylogenetic position of Cannabis sativa. Cannabis belongs to the Cannabaceae, which belong to the order Rosales. Some selected species and their phylogenetic relationship to Cannabis are depicted. Taxonomic groups shaded in blue are inclusive of the taxonomic groups shaded in yellow (e.g. Rosales belong to Fabids, which are eudicots). The timescale at the bottom can be used to infer approximate divergence times.

and Cannabis separated some 120 million years ago, those leading to rice and Cannabis some 130 to 140 million years ago (Figure 3) (Magallón et al., 2015). Among the relatively well-characterized plants that are more closely related to Cannabis are many Rosaceae species (rose family, apple, peach and relatives), for which several well assembled and annotated genomes exist (Aranzana et al., 2019; Zhang et al., 2019), the Cucurbitaceae (cucumber, pumpkin and relatives), which serve as an important model for sex determination and sex expression (Li et al., 2019; Schilling et al., 2020a; Zheng et al., 2019) and Fabaceae (bean family) for flowering time regulation (Cao et al., 2017; Schmutz et al., 2010) .

Cannabis sativa itself is phenotypically extremely diverse. Cannabis plants vary in numerous traits including height, leaf shape, photoperiod response, tetrahydrocannabinol (THC) and cannabidiol (CBD) content, plant architecture and sex expression (Clarke and Merlin, 2016; Grassi and McPartland, 2017; Raman et al., 2017; Schilling et al., 2020b). The dioecy of many Cannabis lines and thus the relatively high levels of heterozygosity further contribute to the fact that even within one cultivar the phenotypic diversity can be substantial (our unpublished observations).

For breeders and farmers, the high level of genetic and phenotypic diversity can be problematic, as a crop is usually best to handle when it possesses a high degree of uniformity in the field. However, at the same time, the existing diversity can be harnessed by breeders to produce new lines for a multitude of different purposes. For plant genetics research, the phenotypic and genetic diversity is a gold mine, as it provides the possibility to study the genetic basis of many traits in Cannabis. Some developments in this arena are outlined in the subsequent chapters, but many more are sure to come. 


\section{Evermore complex: The genetics of phytocannabinoid biosynthesis}

One of the commercially most interesting and valuable products that can be generated from Cannabis plants are phytocannabinoids. We use the term phytocannabinoids here for plant-derived cannabinoids, and to distinguish them from synthetic cannabinoids or those produced by the human endocannabinoid system. Phytocannabinoids are of great interest for medical applications (see chapter 8 for a detailed discussion) as well as commercial exploitations for recreational use. Hence, one of the major breeding goals involves the accurate prediction and targeted manipulation of phytocannabinoid profiles to ensure the optimal combination of active components in plant extracts (see entourage effect chapter 8) or legal compliance for non-psychoactive products.

While there are over 100 different phytocannabinoids described (Pertwee, 2014), three phytocannabinoids are usually at the centre of attention from a medical and commercial perspective: cannabigerol (CBG), cannabidiol (CBD) and tetrahydrocannabinol acid (THC) (Figure 4). Cannabis itself synthesizes phytocannabinoids in the carboxylated form with a carboxylic acid group, i.e. as CBGA, CBDA and THCA. However, to be active in the human endocannabinoid system, phytocannabinoids need to be consumed in their decarboxylated forms, which are usually generated by high-temperature treatment (for example during smoking) (Moreno-Sanz, 2016). Phytocannabinoids are predominantly produced in female inflorescences, more precisely they are secreted from trichomes of perigonal bracts, subtending flowers, and leaves ('sugar leaves') within inflorescences. However, in lower concentrations, phytocannabinoids can also be detected in vegetative leaves at certain times during the growth period (Aizpurua-Olaizola et al., 2016).

Among all phytocannabinoids, THC is the major psychotropic one. However, chemically all molecules mentioned above are very similar in structure and are produced from the same precursor molecules (Figure 4). CBDA and THCA are biochemically synthesized by two closely related enzymes, CBDA and THCA synthase (Shoyama et al., 2012; Taura et al., 1996). CBDA and THCA are both synthesized from CBGA, while CBGA is synthesized from two non-cannabinoids, olivetolic acid and geranyl pyrophosphate by a prenyltransferase (Fellermeier and Zenk, 1998)(Figure 4). Cannabichromenic acid (CBCA) synthase converts CBGA to CBCA (Morimoto et al., 1997) and is closely related to THCA and CBDA synthase (Figure 5), but the CBCA content of most mature Cannabis flowers is low (de Meijer et al., 2009a). Interestingly, CBDA synthase-like genes have been found in other plants and fungi (Aryal et al., 2019; Vergara et al., 2019).

Cannabis plants can have very high levels of phytocannabinoids or close to no phytocannabinoids at all, or anything in between (Aizpurua-Olaizola et al., 2016; de Meijer et al., 2009a). This has stipulated the description of different chemotypes that are characterized by their distinct phytocannabinoid profiles. The chemotypes are a very useful concept for chemical classifications and for breeding programmes. It should be kept in mind, however, that they do not necessarily constitute a phylogenetic classification based on evolutionary relationships (de Meijer et al., 2009b; Small and Beckstead, 1973). Cannabis plants can roughly be categorized into five different 'chemotypes' (Figure 4). Plants of chemotype I (short 'type I') produce high levels of THCA and only low levels of CBDA and CBGA (Small and Beckstead, 1973). This means the ratio of THCA/CBDA is much larger than 1. In type II Cannabis plants THCA and CBDA are both produced in approximately equal amounts (Small and Beckstead, 1973). Both, type I and type II plants, are usually classified as 'marijuana' and can underlie strong regulations, depending on the country or jurisdiction. These plants are bred to produce up to $20 \%$ of their dry mass as phytocannabinoids.

In contrast, type III plants have high CBDA levels and low to very low amounts of THCA.

Chemotype IV and V refer to Cannabis plants which have CBGA as their dominant phytocannabinoid or very low levels of phytocannabinoids overall, respectively (de Meijer et al., 2009a; de Meijer and Hammond, 2005)(Figure 4).

In addition to the five different chemotypes, also the hemp-marijuana distinction is used to characterize different Cannabis plants (Figure 4). If the THC/THCA content in the dry flower mass is below $0.2-1 \%$, these 


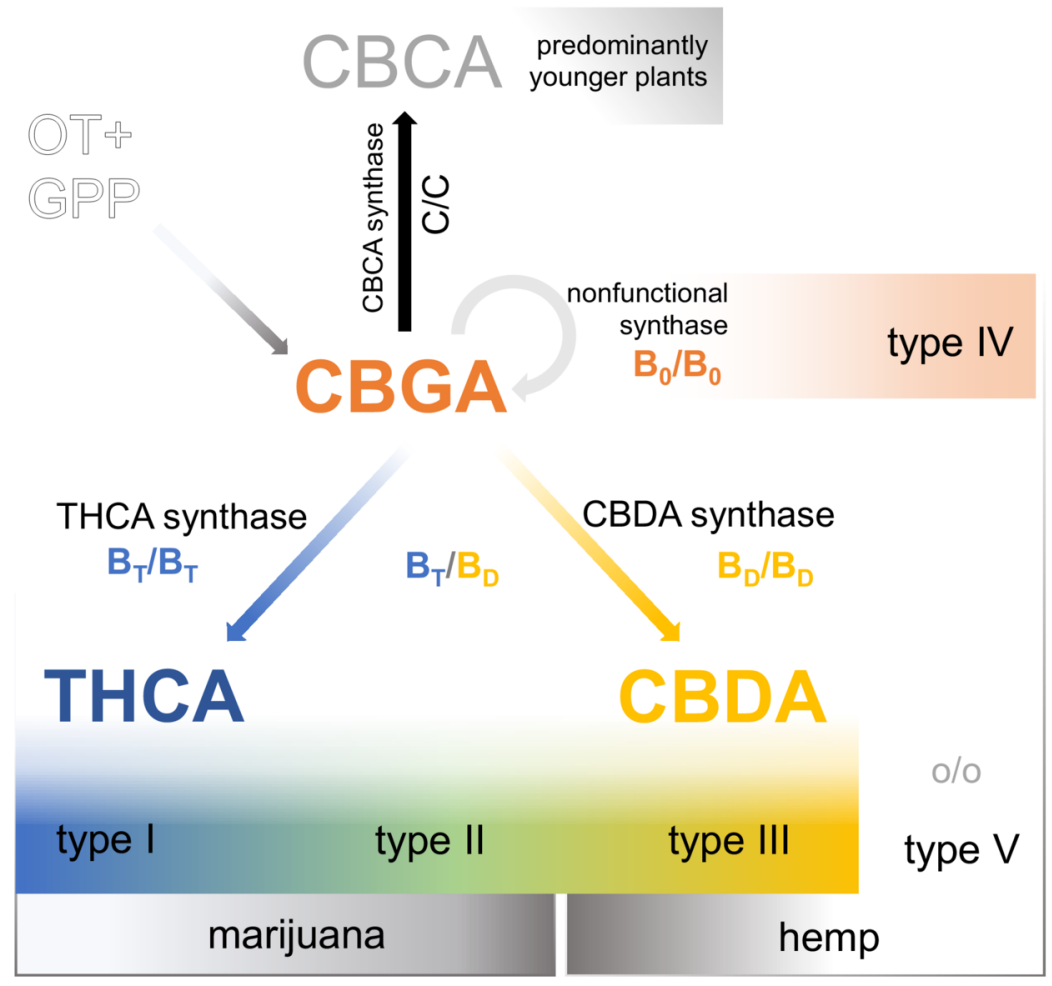

Figure 4: Phytocannabinoids, synthases, genotypes and chemotypes of Cannabis. Phytocannabinoids are synthesised via a multi-step pathway involving different enzymes. The precursor cannabigerolic acid (CBGA) is first synthesised by a prenyltransferase from the precursor molecules geranyl pyrophosphate (GPP) and olivetolic acid (OA). CBGA is metabolised into tetrahydrocannabinolic acid (THCA) via THCA synthase, into cannabidiolic acid (CBDA) via CBDA synthase or cannabichromenic acid (CBCA) via CBCA synthase. The different synthases are encoded by the $\mathrm{B}_{\mathrm{T}}$ (encoding for an active THCA synthase) and $\mathrm{BD}$ (encoding for an active CBDA synthase) loci. $\mathrm{B}_{\mathrm{T}} / \mathrm{B}_{\mathrm{T}}$ plants produce mainly THCA (chemotype I), while $\mathrm{B}_{\mathrm{D}} / \mathrm{B}_{\mathrm{D}}$ plants produce predominantly CBDA (chemotype III). Presence of $\mathrm{B}_{\mathrm{T}}$ and $\mathrm{B}_{\mathrm{D}}$ results in chemotype II (THCA and CBDA intermediate). B0indicates that only non-functional THCA and CBDA synthases are present, which results in the accumulation of CBGA (chemotype IV). Cannabis varieties with very low overall levels of cannabinoids are categorized chemotype $\mathrm{V}$, which is caused by a homozygous recessive allele of locus $\mathrm{O}$. To complicate matters further, there is also a locus $\mathrm{C}$, which is encoding for CBCA synthase. However, in almost all varieties, CBCA is only produced in young immature flowers. Chemotypes I and II can be considered marijuana, while the other low-THC chemotypes can be considered hemp varieties of Cannabis.

plants are usually categorized as hemp, above that as marijuana (depending on the jurisdiction this threshold can vary) (Brunetti et al., 2020; Mead, 2017). The differentiation between hemp and marijuana can typically also be drawn genetically, with hemp and marijuana varieties forming two genetically distinct populations (Sawler et al., 2015). Further, hemp and marijuana can be phenotypically quite distinct with marijuana plants generally being bushier and with a dense set of inflorescences while hemp plants tend to be taller, less branched and with less dense flower structures. However, there are also plants with low THC/THCA content (type III) which strongly resemble marijuana in overall plant and inflorescence architecture (Grassa et al., 
2018). Hence, the terms hemp and marijuana do not necessarily always refer to distinct genetic populations or phylogenetic categories. As the critical distinction between hemp and marijuana is the THC/THCA content, they can also be considered broader categories of chemotypes.

The underlying genetics of the different chemotypes have been studied in quite some detail in the last two decades (de Meijer et al., 2009a, 2009b, 2003; de Meijer and Hammond, 2005; Pacifico et al., 2006; Toth et al., 2020; Weiblen et al., 2015; Welling et al., 2016). However, the complex nature of the Cannabis genome with its many transposable elements, low complexity regions and high heterozygosity have made a conclusive analysis of the loci controlling phytocannabinoid production challenging (Grassa et al., 2018; Laverty et al., 2019; McKernan et al., 2018).

Different genetic loci had been postulated which determine a plant's chemotype, they are encoding for the different types of synthases: at locus $\mathrm{B}$ two codominant alleles were hypothesized to exist, the allele $\mathrm{B}_{\mathrm{T}}$ encodes for the THCA synthase, $B_{D}$ for the CBDA synthase (Figure 4)(de Meijer et al., 2003). Depending on the presence of either or both loci, the plant will be chemotype $\mathrm{I}\left(\mathrm{B}_{\mathrm{T}} / \mathrm{B}_{\mathrm{T}}\right)$, chemotype II $\left(\mathrm{B}_{\mathrm{T}} / \mathrm{B}_{\mathrm{D}}\right)$ or chemotype III $\left(\mathrm{B}_{\mathrm{D}} / \mathrm{B}_{\mathrm{D}}\right)$ (de Meijer et al., 2003; Toth et al., 2020; Welling et al., 2016). Additionally, non-functional alleles of the synthase gene $\left(\mathrm{B}_{0}\right)$ are predicted to be associated with chemotype IV, where neither CBDA nor THCA are produced and the precursor, CBGA, accumulates (Figure 4) (de Meijer and Hammond, 2005; Onofri et al., 2015; Welling et al., 2016).

Further, according to this model, CBCA synthase is encoded by an independent locus (C) while another independent locus $(\mathrm{O})$ is relevant for precursor production, with a knockout resulting in overall minimal phytocannabinoid levels (Figure 4) (de Meijer et al., 2009a, 2009b).

The genetic basis of the chemotypes was analysed in detail by producing a cross between high-THC Purple Kush (chemotype I) and low-THC Finola (chemotype III). This resulted in an F1 generation of mainly type II plants, producing both, THCA as well as CBDA (Weiblen et al., 2015). This confirmed earlier findings of crosses between type I and type II plants, resulting in intermediate type II individuals (de Meijer et al., 2003). The segregation pattern of phytocannabinoid profiles in the F2 generation pointed towards a Mendelian inheritance pattern: type I, type II and type III plants were all observed in the F2 generation with the expected distribution of 1:2:1 (de Meijer et al., 2003; Weiblen et al., 2015). A correlation of the expression of either THCA or CDBA synthase with the respective chemotype was also observed and the THCAS/CBDAS locus could be mapped (Weiblen et al., 2015).

However, although these findings were consistent with the idea of codominant alleles at one single locus, it became apparent that the situation is more complex (Grassa et al., 2018; Laverty et al., 2019; Weiblen et al., 2015). New draft genomes generated with third-generation sequencing technology indicated that the THCA and CBDA synthases do not seem to be encoded by alleles of one and the same gene, but rather by distinct loci in marijuana and hemp, respectively, without a clear counterpart in the other genome (Grassa et al., 2018; Laverty et al., 2019). Sequencing of the hemp cultivar 'Finola' and the marijuana cultivar 'Purple Kush' indicates that a functional CBDA synthase gene is present only in in the 'Finola' genome while the 'Purple Kush' genome only encodes for a functional THCA synthase (Laverty et al., 2019). While mapping to approximately the same region in both genomes, the DNA sequences surrounding the respective synthase genes are drastically different from each other. Further, a low albeit still detectable recombination rate between the two loci supports the notion that they are genetically distinct (Laverty et al., 2019). The sequencing of a different Cannabis variety ('CBDRx'), which is a chemotype III hemp-marijuana hybrid revealed an even more complex genomic arrangement with a number of pseudo- and functional synthase genes in three different cassettes on the same chromosome (Figure 5) (Grassa et al., 2018).

The CBDA and THCA synthase genes themselves seem to be embedded in cassettes of multiple tandem duplications of putatively non-functional synthase genes, which are regularly interspersed with long terminal repeat (LTR) retrotransposons, making the assembly and analysis of these loci even more challenging (Figure 5) (Grassa et al., 2018; Laverty et al., 2019). This is also the reason why these complex loci could not be resolved in the first published Cannabis genome, which relied on short-read sequencing data (van Bakel et 
al., 2011). This genomic constitution, where the difference between marijuana and hemp comes down to a large structural variation is, if true, very unusual. Hence, the aforementioned locus "B" with its different alleles might look very different from what was previously assumed to be simple isoforms of a single gene.

The complexity of phytocannabinoid synthases does not end there, though. Copy number variation of CBDA and THCA synthase genes might be involved in phytocannabinoid level and composition (Vergara et al., 2019) and most likely, the number of synthase (pseudo)genes might be different for each cultivar sequenced (Grassa et al., 2018; Laverty et al., 2019; McKernan et al., 2020).

High throughput assays for $\mathrm{B}_{\mathrm{T}}$ and $\mathrm{B}_{\mathrm{D}}$ markers have been developed and show that many plants actually contain both loci (Cascini et al., 2019; McKernan et al., 2020; Toth et al., 2020). Moreover, many $\mathrm{B}_{\mathrm{D}} / \mathrm{B}_{\mathrm{D}}$ plants, especially those with higher CBDA levels, have THCA levels of above $0.3 \%$ of dry flower mass, despite the absence of a functional $\mathrm{B}_{\mathrm{T}}$ allele (Toth et al., 2020). This residual THCA is probably at least to some extent a by-product of the CBDA synthase itself. The THCA and CBDA synthase have a relatively high sequence similarity ( $83.85 \%$, Figure 5 ) and process the same precursor molecule, CBGA (Figure 4). In vitro studies have shown that the CBDA synthase produced CBDA and THCA at roughly a ratio of 20:1 (Zirpel et al., 2018). This is similar to ratios observed in planta in high-CBD hemp varieties as well (Toth et al., 2020; Weiblen et al., 2015). This potentially results in the problem that, if CBDA production is increased, THCA also increases as a by-product, even if plants do not express a functional THCA synthase. Cannabis varieties with very high CBD levels may thus be at risk of exceeding legal THC thresholds.

Understanding the exact genetics underlying the different chemotypes will be important for future targeted breeding approaches. Tight restrictions across the world make it difficult for farmers to grow chemotype III, IV and V varieties, because the presence of residual THC creates regulatory problems and uncertainties. Especially type III plants often have THCA/THC levels slightly above the legal THC limit (AizpuruaOlaizola et al., 2016; Toth et al., 2020). Hence, one important breeding goal is going to be the generation of zero-THC lines which still produce high levels of CBD in the range of 15 to $20 \%$ of dry flower mass. Whether this is possible to achieve is difficult to say, since even in the absence of a THCA synthase, CBDA synthases produce THCA as a by-product (Toth et al., 2020; Zirpel et al., 2018). This will, therefore, require identification of a CBDA synthase that does produce only very low or no amounts of THCA.In vitro experiments show that point mutations can alter the amount of by-products (Zirpel et al., 2018). Natural variation in synthase genes exists and have been linked to altered phytocannabinoid compositions (Onofri et al., 2015). Hence, naturally occurring or artificially generated CBDA synthase varieties could be used for targeted breeding in this direction.

In addition, Cannabis varieties used for fibre or seed production could be selectively bred and genotyped to have $0 \%$ overall phytocannabinoids (chemotype $\mathrm{V}$ ), as currently even the farming of these kinds of varieties is heavily restricted in many countries.

Other phytocannabinoids like $\mathrm{CBG}(\mathrm{A})$ and $\mathrm{CBC}(\mathrm{A})$ as well as the manifold variants of terpenes produced in Cannabis flowers are increasingly coming into focus in the medical research fields (reviewed in Booth and Bohlmann, 2019; Deiana, 2017; Pollastro et al., 2018), hence generating lines with specific phytocannabinoid profiles might be of interest in further research. 

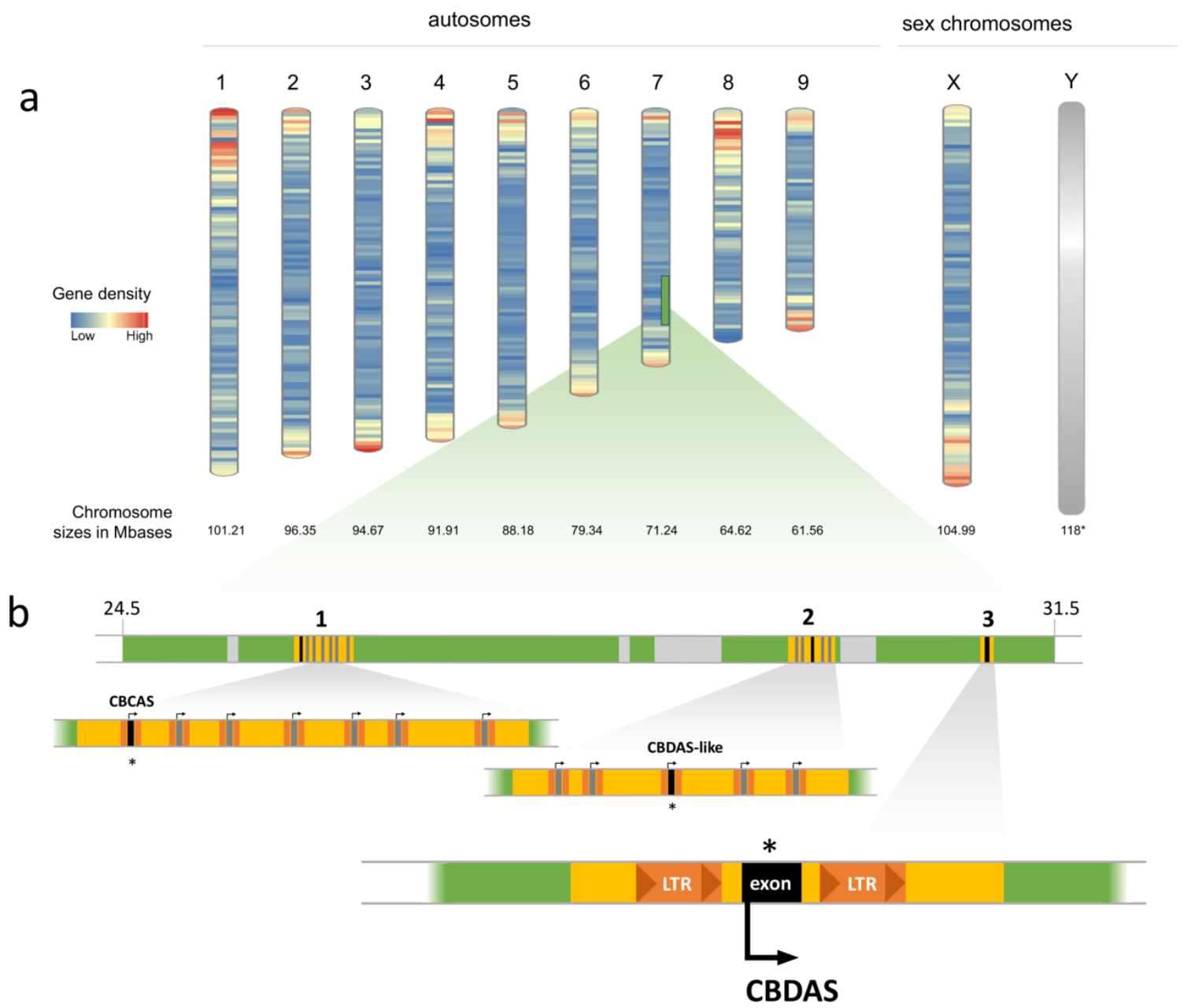

C

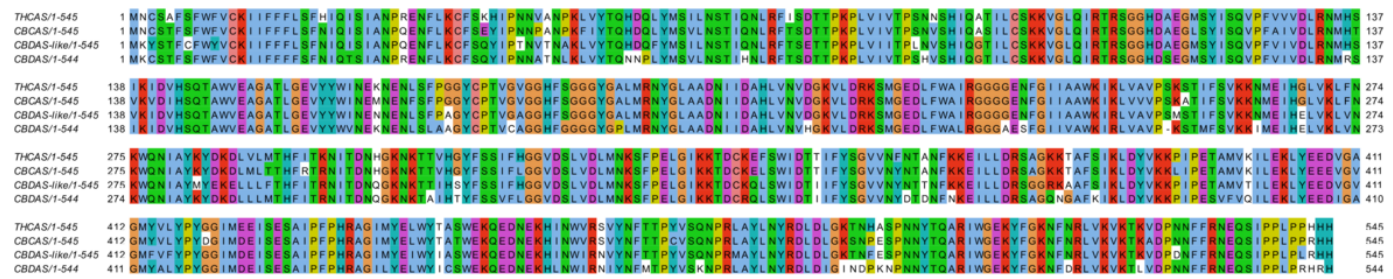

Figure 5: Genetic location of genes encoding cannabinoid synthases in Cannabis. Preliminary analysis of location and structure of the genes encoding CBCA synthase, CBDA synthase and multiple cannabinoid-synthase-like pseudogene copies in the hemp-marijuana hybrid cultivar 'CBDRx' (Grassa et al., 2018), which is the reference genome for Cannabis sativa. The genome has 9 pairs of autosomes and one pair of sex chromosomes, with the main locus of cannabinoid synthases located on chromosome 7 (green box, revised chromosome numbering (NCBI)) (a). Three different cassettes (1, 2 and 3, yellow regions with stripes) have been identified and mapped to a region on chromosome 7 between 24.5 and $31.5 \mathrm{Mb}$ (b). The exact chromosomal arrangement is not clear, since the assembly contains gaps in between the different cassettes (grey boxes). Three cannabinoid synthase genes appear to have a full coding sequence: a CBCA synthase, the CBDA synthase and a CBDA-like synthase (*, black), while the other copies appear nonfunctional (grey). All sequences, including pseudogenes, have unique expression data associated with them (arrows, NBCI genome browser, unique raw reads). The synthases are encoded by one single exon (black) and surrounded by long terminal repeat retrotransposons (orange). Functional CBDA and THCA synthase share $83.86 \%$ protein sequence similarity, while similarity amongst the other sequences ranges from 82 to 92 $\%$ (c). The CBDRx genome does not contain a functional THCAS, the sequence was acquired from uniprot (Q8GTB6). Gene density in (a) shows the ratio of the number of genes per million base pairs, calculated and plotted as an ideogram using RIdeogram (Hao et al., 2020). Gene annotation for the Y chromosome is not readily available, but genes are present (Prentout et al., 2020; McKernan et al., 2020). The CBDRx genome was derived from a female individual (Grassa et al., 2018), the size of the Y $\left(^{*}\right)$ chromosome was approximated from McKernan et al., 2020. 


\section{A hairy topic: Flower development and morphology in Cannabis}

The flower is the reproductive structure of flowering plants (angiosperms), which represent one of the most successful and diverse groups of organisms on this planet (Krizek and Fletcher, 2005). While the characteristic shape of the Cannabis leaf is often used as a symbol for the whole plant, Cannabis female flowers are of particular interest because they are the main site of production of pharmacologically active compounds (phytocannabinoids) (Spitzer-Rimon et al., 2019). Understanding the morphology of Cannabis flowers and their developmental genetics is therefore especially important.

The typical angiosperm flower consists of four different organ types, which are organized in concentric whorls: sepals, petals, stamens and carpels (Endress, 1992; Krizek and Fletcher, 2005). Sepals are in the outermost whorl and usually green and leaflike in appearance. Petals are in the second whorl and often coloured to attract pollinators. Petals together with sepals are termed the perianth and constitute the non-reproductive part of a flower. Stamens are typically located in the third floral whorl. They are the male reproductive organs and are composed of an anther and a filament. The anthers grow on top of the stalk-like filaments and are the site of pollen production. Finally, carpels develop in the fourth and central whorl of a typical flower. Carpels are the reproductive organs that contain an ovary inside which ovules develop. The tip of the carpel, the stigma, receives the pollen. The style connects the stigma to the ovary (Becker, 2020; Endress, 1992; Krizek and Fletcher, 2005).

Notably, the number, arrangement, and morphology of the floral organs varies substantially between different species of flowering plants (Endress, 2011; Theissen and Melzer, 2007). Most flowers contain, as described above, both carpels and stamens, and are therefore termed bisexual flowers (Renner, 2014). However some 15 $\%$ of flowering plant species are monoecious or dioecious and have unisexual flowers that develop only stamens or carpels (Renner, 2014). In dioecious plants, female and male flowers develop on separate individuals. In contrast, in monoecious plants male and female flowers develop on the same individual (Renner, 2014).

Cannabis is primarily dioecious (Moliterni et al., 2004). The male Cannabis flower is green-yellow in appearance and has a perianth of five sepals, while petals are completely absent. Further, an individual male flower contains five free stamens, and no female reproductive organs (Figure 6a and b) (Leme et al., 2020; Spitzer-Rimon et al., 2019).

On the other hand, the female flower is enclosed within a green leaflike perigonal bract. The perigonal bract is sometimes also described as a sepal, but morphological studies agree that it is a bract (Leme et al., 2020; Spitzer-Rimon et al., 2019). As such, it is not strictly a part of the flower. Between the perigonal bract and the carpel is a membranous and hyaline perianth which tightly embraces the ovary (Leme et al., 2020; Reed, 1914; Spitzer-Rimon et al., 2019). It is worth noting that this inconspicuous perianth sometimes is not mentioned in the structure of female Cannabis flowers or is considered missing as it is not visible from the outside of the flower. Most likely, these membranous structures are homologous to sepals (Leme et al., 2020). At the top of the ovary are two filamentous styles. The stigma is brush-like and has epidermal cells elongated into hair-like projections (Reed, 1914; Lemeet al., 2020) (Figure 6c and d).

The commercially interesting phytocannabinoids and terpenes are predominantly produced on the perigonal bracts of female flowers, more specifically in glandular trichomes that cover those bracts. Glandular trichomes can be categorized into sessile, stalked and bulbous trichomes (Hammond and Mahlberg, 1973), with bulbous trichomes being metabolically less active (Livingston et al., 2020). Cannabis plants also have non-glandular trichomes: hair-like uni- or multicellular trichomes which protect them from biotic and abiotic stresses (Andre et al., 2016; Dayanandan and Kaufman, 1976). However, glandular trichomes are the main site of phytocannabinoid synthesis (Furr and Mahlberg, 1981).

Because phytocannabinoids are cytotoxic in higher concentrations, they have to be secreted and are not stored within cellular compartments. Phytocannabinoids along with other secondary metabolites are secreted from glandular trichomes with a globose head-like structure (Figure 7). This head is formed by an enlarged 


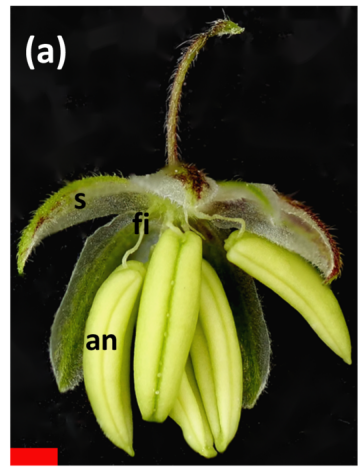

(b)
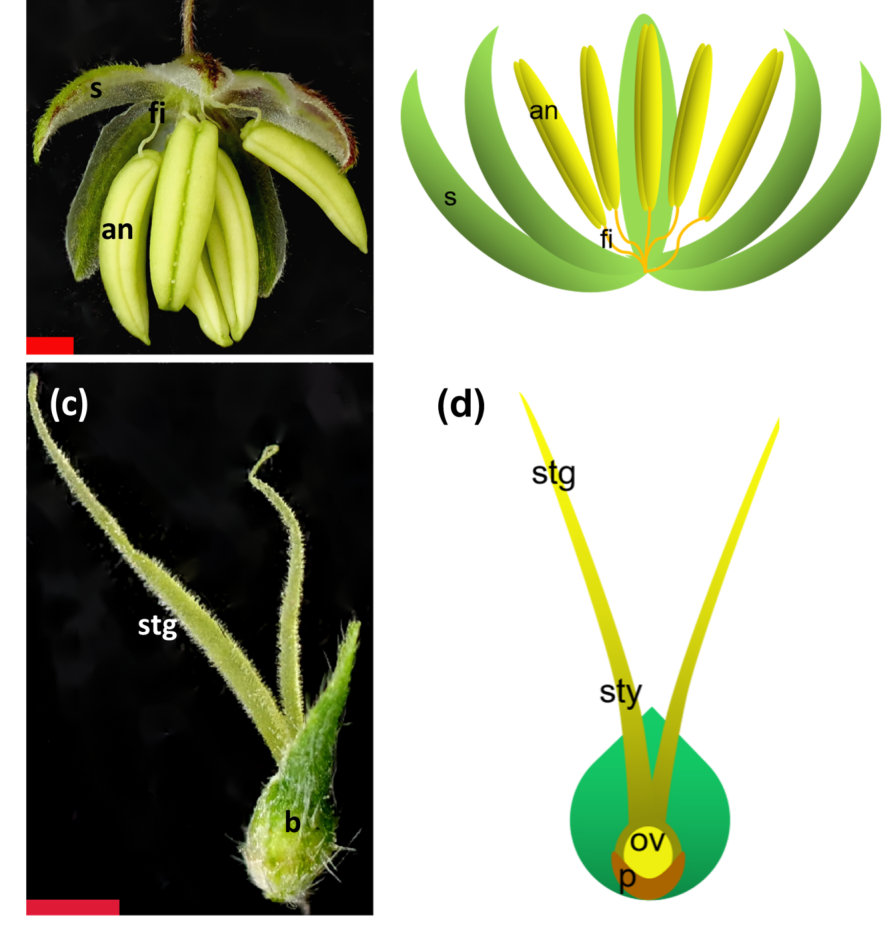

(d)

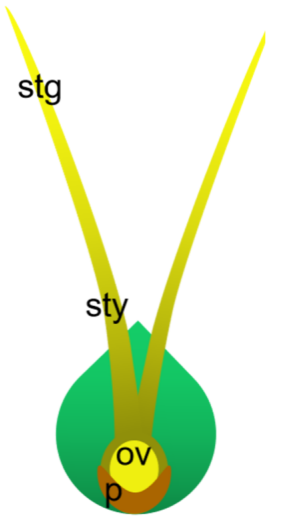

Figure 6: Mature flowers of Cannabis sativa. Male Cannabis flowers have sepals and stamens (a) while female Cannabis flowers consist of two carpels enclosed by a perigonal bract (c). Schematic depiction of the structure of Cannabis male flower (b) and female flower (d). The bar $=1 \mathrm{~mm}$. an, anther; b, perigonal bract; fi, filament; s, sepal; ov, ovary; p., perianth; stg, stigma; sty, style.

secretory cavity which is surrounded by a culticule that encapsulates the secreted secondary metabolites (Hammond and Mahlberg, 1973). At the base of the head is a layer of secretory cells (Kim and Mahlberg, 1991; Livingston et al., 2020). The head can be sessile, directly on the epidermis and often be found on vegetative leaves (sessile trichomes), or pre-stalked or stalked with the head being elevated above the epidermis (pre-stalked and stalked trichomes), which are mainly found on female inflorescences (Kim and Mahlberg, 1991; Livingston et al., 2020). Additionally, these structures can be distinguished by different levels of autofluorescence, cell numbers as well as phytocannabinoid and terpene profiles (Livingston et al., 2020; Turner et al., 1978). Stalked trichomes seem to be developing from pre-stalked trichomes and contain a terpene profile distinct from true sessile trichomes (Livingston et al., 2020). Transcriptome analysis of floral trichomes of a CBD hemp ('Finola') confirmed high expression levels of genes involved in the synthesis of phytocannabinoids, terpenes and their respective precursor molecules in glandular trichomes, with expression differences between bulbous, sessile, and (pre-)stalked trichomes (Livingston et al., 2020).

It is not clear why predominantly female plants produce glandular trichomes within their inflorescence structures. Illuminating the genetic underpinnings of this sexual dimorphism remains a challenge for further research. Glandular trichomes also develop on male flowers (Leme et al., 2020), albeit at lower density and probably with less phytocannabinoids. Understanding which genetic factors restrict the development 

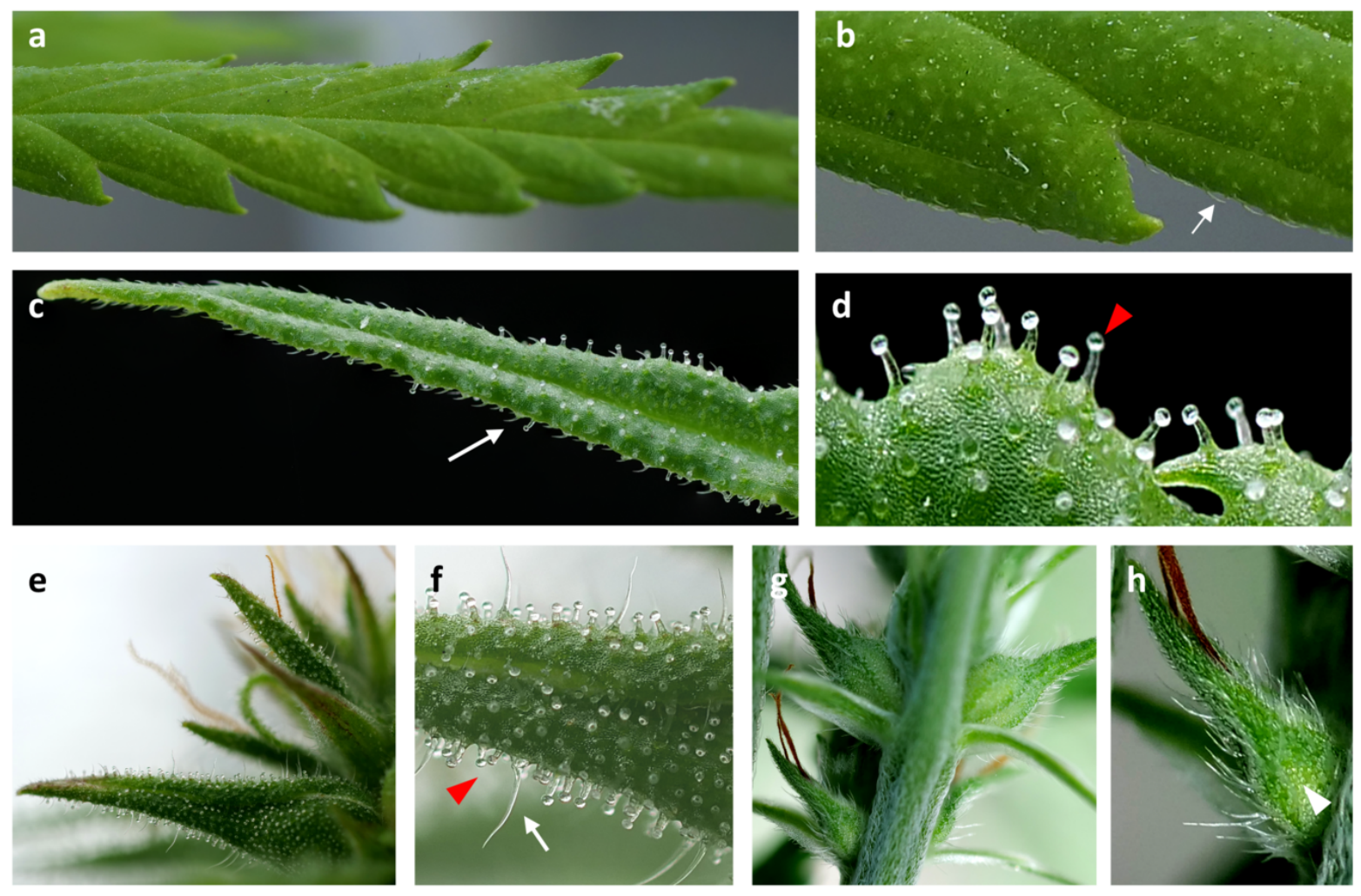

Figure 7: Different types of trichomes on Cannabis sativa hemp varieties. Trichomes can be found on the majority of female Cannabis plant epidermises of the cultivars 'Finola' (a-f) and 'Felina 32' (g, h). Vegetative leaves (a) have mainly non-glandular hair-like trichomes (b). Subtending ("sugar") leaves within an inflorescence (c) do have both, non-glandular trichomes as well as glandular trichomes (d). The perigonal bract of a female 'Finola' flower (e) is covered in glandular stalked trichomes (f), while the majority of trichomes found on the bract of 'Felina 32' flowers (g) are non-glandular and sessile trichomes (h). White arrows: non-glandular trichomes; white arrowheads: sessile trichomes; red arrowheads: stalked trichomes.

of glandular trichomes largely to female inflorescences during flower development would provide a valuable resource for an increase of phytocannabinoid production.

\section{The battle of the sexes: Sex determination in Cannabis}

\subsection{The genetics of sex determination}

The dioecy of Cannabis is genetically controlled (Figure 2). Hemp is diploid $(2 \mathrm{n}=20)$, with nine pairs of autosomes and one pair of sex chromosomes. Female plants are homogametic with XX chromosomes and male plants are heterogametic with an XY sex chromosome pair (Moliterni et al., 2004). Cannabis thus represents a rare case among the flowering plants in which sex chromosomes have been identified (Charlesworth, 2016).

The diploid genome size of female Cannabis plants is estimated to be $1636 \mathrm{Mbp}$, that of a male plant 1683 Mbp by flow cytometry (Sakamoto et al., 1998). The sex chromosomes of Cannabis are the largest in the chromosomal complement, they are estimated to comprise $6.5 \%$ (Y chromosome) and $6.1 \%$ (X chromosome) 
of the total length of the genome (Divashuk et al., 2014). Assuming that those estimates roughly correspond to length in base pairs (which is most certainly an oversimplification), the $\mathrm{X}$ chromosome would be 102.7 Mbp in size, and the $\mathrm{Y}$ chromosome 109.4 Mbp. This is close to the size of the $\mathrm{X}$ and Y chromosomes as determined by genome sequencing (Figure 5, Supplementary Table 1). However, the flow cytometry results mentioned above (Sakamoto et al., 1998) indicate that the $\mathrm{Y}$ chromosome is $47 \mathrm{Mb}$ larger than the $\mathrm{X}$ chromosome $(1683 \mathrm{Mb}-1636 \mathrm{Mb}=47 \mathrm{Mb})$. Flow cytometry analyses of other Cannabis varieties yielded very similar results (Faux et al., 2014). It is not clear where the discrepancy between genome sequencing and flow cytometry measurements is coming from. Structural genomic variations between different Cannabis plants as well as problems in assembling the sex chromosomes may both play a role.

Detailed analyses of the sex chromosomes revealed that the pseudo-autosomal region on the $\mathrm{X}$ chromosome (i.e. the region still recombining with the $\mathrm{Y}$ chromosome) is ca. $30 \mathrm{Mb}$ in size, whereas the $\mathrm{X}$-specific region (which is not recombining with the $\mathrm{Y}$ chromosome) is ca. $75 \mathrm{Mbp}$ in size (Prentout et al., 2020). Prentout et al. also identified ca. 500 sex-linked genes, i.e. alleles that are inherited in a sex-linked fashion (e.g. only from father to daughter, not from father to son for X-hemizygous alleles). It will be especially interesting to analyse the $\mathrm{X}$ chromosome alleles that have no $\mathrm{Y}$ chromosome counterpart in detail in the future, as they may contribute to sex determination.

Whereas gene density on the $\mathrm{X}$ chromosome appears to be similar to autosomes, about $70 \%$ of the genes on the Y chromosomes have been estimated to be lost (Prentout et al., 2020). One explanation for the relatively large size of the $\mathrm{Y}$ chromosome despite substantial gene losses seems to be the accumulation of transposons and other repetitive elements (Sakamoto et al., 2000). Prevalence of transposable elements on the Y chromosome might also help to explain difficulties in chromosome assembly and discrepancies in size estimates.

Despite progress in identification and sequencing the sex chromosomes of Cannabis, not much is known about the molecular circuits involved in sex determination. Some confusion exists as to what the genetic 'mode' of sex determination is (Kovalchuk et al., 2020; Vergara et al., 2016). Some reports suggest the mode is similar to humans and other mammals, where the presence or absence of the Y chromosome determines the sex: humans carrying a Y chromosome are almost always phenotypically male, those without a $\mathrm{Y}$ chromosome are female, with autosomes or extra $\mathrm{X}$ chromosomes bearing little consequence on the sex determination (Gamble and Zarkower, 2012; Sakamoto et al., 1998). An alternative view is that the X chromosome to autosome ratio determines the sex (Westergaard, 1958). This would be somehow similar to Drosophila, where the number of $\mathrm{X}$ chromosomes determines the sex, with the presence or absence of the $\mathrm{Y}$ chromosome having limited relevance (Gamble and Zarkower, 2012). In this model, the Y chromosome essentially becomes a 'placeholder', lowering the number of X chromosomes.

Experimental evidence supporting the one or the other mode of sex determination is surprisingly scarce. Warmke and Davidson used colchicine to produce tetraploid Cannabis plants (Warmke and Davidson, 1944). Strikingly, a cross between a tetraploid female and a diploid male plant yielded female and femalehermaphrodites, but no male plants, though half of the progeny should have an XXY chromosome constitution (Warmke and Davidson, 1944). This is evidence that the Y chromosome does not play a role as prominent as in humans in sex determination in Cannabis, but that an $\mathrm{X}$ to autosome ratio model for sex determination might apply.

It is important to note that, even if an $\mathrm{X}$ to autosome ratio model applies in Cannabis, this does not mean that the Y chromosome is dispensable for the development of male plants. In Drosophila, the Y chromosome is not involved in sex determination but encodes genes required for male fertility, such that XO flies are phenotypically male but sterile (Gamble and Zarkower, 2012). Likewise, Y chromosome specific genes in Cannabis may well play a critical role in male plant development, even if not involved in bona fide sex determination (McKernan et al., 2020). 


\subsection{Monoecy, dioecy and evolutionary considerations}

To add further complications to the study of sex determination inCannabis , monecious varieties exist, in addition to the canonical dioecious varieties. Monoecious cultivars develop male and female flowers on the same plant and are particularly popular for fibre production (Figure 2). This is because in dioecious varieties, male plants flower earlier than female plants, whereas for monoecious cultivars flowering time is more synchronized, thus facilitating the determination of an optimal harvest time (Faux et al., 2016).

Genetically, monoecious cultivars carry two X chromosomes and no Y chromosome, indicating that the XY sex determination system can be 'leaky' in Cannabis(Faux et al., 2014; Razumova et al., 2016). In addition, the monoecious cultivars express 'femaleness' and 'maleness' to different degrees, i.e. the ratio of female to male flowers one plant develops differs between cultivars but also between different environmental conditions (Faux et al., 2014). At least some genetic loci relevant for the sex expression in monoeciousCannabis plants seem to be located on the X chromosome (Faux et al., 2016). It will be interesting to see whether the distinction between dioecious and monoecious cultivars and between different degrees of sex expression (femaleness or maleness) in monoecious cultivars can be traced back to the same molecular circuits.

From an evolutionary point of view, the sexual system in the entire family of Cannabaceae is complex. In contrast to angiosperms in general, some $85 \%$ of which are bisexual (Renner, 2014), true bisexual flowers are conspicuously rare in Cannabaceae (Yang et al., 2013). Several shifts of the sex determination system occurred in the Cannabaceae, and ancestral character state reconstructions indicate that monoecy, or, with a lesser likelihood, dioecy, is the ancestral state in the family (Yang et al., 2013). Interestingly, one of the closest relatives ofCannabis sativa, Humulus lupulus (common hop), is dioecious with an XY sex determination system in which the $\mathrm{X}$ to autosome ratio determines the sex (Parker and Clark, 1991). This may be taken as additional evidence that also Cannabis has an $\mathrm{X}$ to autosome ratio sex determination system. However, it should be kept in mind that that Humulus andCannabis may have separated some 25 million years ago (Jin et al., 2020) and that sex determination systems can frequently and rapidly change during evolution (Bachtrog et al., 2014). Nevertheless, available data on the sex determination systems in Cannabaceae as a whole may be taken as indication that all family members share an ancestral molecular mechanism for sex determination, but that this mechanism is relatively labile and manifests differently in different species.

\subsection{Hormonal and environmental factors affection sex determination}

Beyond genetic consideration, the sex expression in Cannabis can be shaped by environmental factors. It is well established that silver can be used to induce the formation of male flowers on female plants (Mohan Ram and Sett, 1982). This treatment is used by breeders to self female dioecious plants, which results in offspring with exclusively XX sex chromosomes (Clarke and Merlin, 2016). Because all of the progeny from such a selfed plant will be female, the 'feminized' seeds produced that way are usually much more valuable than conventionally produced seeds (Small, 2015). Silver is a known ethylene inhibitor, and there is also evidence that ethylene induces the development of female flowers on male plants (McDaniel and Binder, 2012; Ram and Jaiswal, 1970). Together, this therefore suggests that ethylene is involved in controlling the sex expression in Cannabis .

In addition to ethylene, also other phytohormones have been shown to be capable of altering the sex expression in Cannabis(Ainsworth, 2000) . Auxin, for example, can lead to the development of female flowers on male plants. Indeed, male flower development can be entirely repressed by auxin treatment (Heslop-Harrison, 1956). Cytokinin also has a feminizing effect (Chailakhyan and Timiriazev, 1979), whereas gibberellic acid triggers the formation of male flowers on female plants (Ram and Jaiswal, 1972). Together, the hormonal effects are very similar to what is observed for sex expression in the well analysed Cucurbitaceae family (Li et al., 2019; Pawełkowicz et al., 2019; Schilling et al., 2020a). Cucurbitaceae are only distantly related to Cannabaceae, the two lineages separated more than 100 million years ago (Figure 3) (Magallón et al., 2015). Also, many of the studied Cucurbitaceae are monoecious and thus do not possess sex chromosomes (Boualem et al., 2015). Nevertheless, we hypothesize that similar developmental genetic pathways may have 
been co-opted to control sex expression in Cannabis and the Cucurbits. Melon, cucumber, pumpkin, and their relatives might thus serve as an excellent model to unravel the molecular intricacies of sex expression and sex determination in Cannabis .

Cannabis is a short-day plant, and flowering is initiated if day length is below $14 \mathrm{~h}$ (see below), but it is well established that sex expression is also affected by day length. It was reported some 100 years ago already that dioecious plants grown under short day conditions only (i.e. without an initial period of long day growth) can develop male as well as female flowers ((Tournois 1911, 1912, as cited by (Heslop-Harrison, 1957), (Schaffner, 1923)). A lot of other environmental factors like nitrogen availability or carbon monoxide also seem to have an influence on sex expression (Freeman et al., 1980; Heslop-Harrison, 1957; Small, 2015). Together, this yields a very complex picture of different environmental factors influencing sex expression in different directions that is far from being completely understood.

In summary, the picture emerges that, although sex determination is genetic, hormonal and environmental influences have a significant effect on sex expression. Because the flowers of female plants are the main source of phytocannabinoids, a more detailed study of the sex determination and sex expression mechanism of Cannabis is one of the main areas of future research. For example, creating male sterility would be very beneficial, as phytocannabinoid production is highest in unpollinated female plants. Studying both sex chromosomes, their gene content as well as the molecular intricacies of the sex determination mechanisms will certainly provide valuable insights for breeders as well as researchers.

\section{Timing is everything - the complex network of floral initiation and hints for Cannabis}

\subsection{The evolutionary and developmental importance of flowering time}

Flowering time control is essential for reproductive success (Shim et al., 2017). Mechanisms to control the time of flowering have evolved given the negative consequences of spontaneous floral initiation: Premature flowering could coincide with the absence of pollinators or dispersers, subsequently causing reduced fertilisation rates and deficient seed dispersal. Conversely, if flowering occurs too late the plant may fail to set seed before harsh conditions hit at the end of the growing season (Gaudinier and Blackman, 2020). Moreover, in dioecious species such as Cannabis, the timing of flower emergence is particularly crucial, because if male and female plants do not flower concurrently, pollination cannot occur (Hall et al., 2012). Hence it is evolutionarily beneficial for plants to possess mechanisms to fine-tune their floral initiation (Gaudinier and Blackman, 2020). Analyses in various plant species have demonstrated that flowering time in angiosperms is controlled by internal timekeeping mechanisms as well as environmental signals. Among the major factors controlling flowering time are the plant age, the photoperiod, the circadian clock, ambient temperature, the phytohormone gibberellin and the autonomous pathway (Fornara et al., 2010; Hill and Li, 2016).

The fine-tuning of flowering time is a major goal for plant breeding and crop improvement efforts. Floral transition represents the developmental shift from vegetative to reproductive growth and is a major determinant of yield potential (Jung and Müller, 2009). Alterations in key flowering time genes have been crucial to crop domestication, facilitating the adaptation of crops to local climatic conditions (Gaudinier and Blackman, 2020; Schilling et al., 2018). The success and worldwide expansion of staple crops like wheat and rice can partly be attributed to natural variation in flowering time genes, which enabled local adaptation for cultivation at a wide range of latitudes (Hill and Li, 2016; Langer et al., 2014). As a quantitative short-day plant, Cannabis flowering time is particularly determined by the photoperiod. Under long-days Cannabis remains vegetative and flowering is only induced when a number of short-day photoperiods have passed. Therefore, in order to cultivate Cannabis at new lines of latitude (for example in Ireland where summer daylengths can be over $17 \mathrm{~h}$ ), the adjustment of flowering time genes can be advantageous. Consequently, comprehensive characterisation of the Cannabis flowering time pathways is crucial to the integration of this 
crop species into modern agriculture.

\subsection{Flowering time control in Cannabis - what we know so far}

Cannabis has the potential to be a sustainable multipurpose crop. For virtually all applications of Cannabis , a better understanding of the genetic factors controlling flowering time would be highly beneficial (Figure 1). The reasons are evident when the flowers or seeds are the main agricultural product, such as hemp oil from seeds or CBD production from flowers. But flowering time also determines the crop purpose in more general terms, with later flowering varieties favouring vegetative stem growth thus suiting fibre production and earlier varieties displaying enhanced flower/seed yield (Salentijn et al., 2019). The interactions between flowering time and fibre quality are complex (reviewed in Salentijn et al., 2019) and the developmental stage at harvest has major implications for fibre quality. Additionally, a better understanding of flowering time is important to generate varieties that are adapted to local climatic and photoperiod conditions.

While various environmental signals including temperature (Amaducci et al., 2012, 2008; Cosentino et al., 2012; Nelson, 1944) prompt floral initiation, Cannabis is particularly sensitive to changes in photoperiod (Hall et al., 2012; Salentijn et al., 2019). In as early as 1912 it was observed that flower induction in Cannabis is influenced by the photoperiod (Tournois 1912 (cited by Heslop-Harrison, 1957)). Cannabis is a facultative short-day plant (Salentijn et al., 2019). This means that while plants will typically flower eventually under long-day conditions, flowering occurs faster in short-day conditions i.e. by experiencing a sequence of days each with a minimum uninterrupted period of darkness. Cultivar-specific variation for the photoperiod at which flowering is induced has been reported, with the optimal photoperiod ranging from 9 to $14 \mathrm{~h}$ (Lisson et al., 2000 and references cited therein).

A related question is how many consecutive short days are required to induce flowering. Borthwick and Scully (1954) reported two weeks of a short photoperiod induced flowering in 3-5-week-old plants, and the greater the plant age at the time of switching to short-day the faster the floral transition. Furthermore, Potter (2014) stated flowers can be visible one week after the reduction in day length. Clearly, more research is needed in this area, in particular to explore the variation between cultivars for this trait.

Given that most individuals eventually flower under non-inductive photoperiodic conditions, a more detailed analysis of the age-related and the autonomous pathway and their influence on flowering time is warranted. In addition, it would be especially interesting to observe stressors that can accelerate flowering under noninductive photoperiodic conditions, and detect whether the same signalling pathways are at play when individuals eventually flower under long days (Takeno, 2016).

\subsection{Model plants and candidate genes: Arabidopsis, soybean and the hunt for flowering time genes in Cannabis}

While important efforts to determine the environmental stimuli impacting floral induction in Cannabis have been undertaken, the genetic pathways and loci underlying the environmental responsiveness still require elucidation (Salentijn et al., 2019). Huge diversity exists for flowering time in Cannabis with phenotypes generally categorised as early-, mid- or late-flowering (Salentijn et al., 2019). Furthermore, photoperiodinsensitive (also known as day-neutral or auto-flowering) cultivars exist (Small, 2018). A recent study suggests that female floral initiation occurs independently of the photoperiod in some Cannabis cultivars, while in others shorter photoperiods were required for flower maturation and development (Spitzer-Rimon et al., 2019). Further research is required to substantiate the molecular basis of those observations, and research on model plants may serve as an important primer to understand the gene regulatory network controlling flowering time in Cannabis.

Among the model plant species for which comprehensive analyses of flowering time have been conducted are the long-day plantArabidopsis thaliana and the short-day plant Oryza sativa(rice). In Arabidopsis, the complex flowering time network is well-characterised with several pathways described including the vernalisation, 
autonomous, photoperiod, circadian clock, age, ambient temperature and gibberellin pathways (Blümel et al., 2015). One of the key integrators of floral inductive signals in Arabidopsis is FLOWERING LOCUS T $(F T)$, the protein product of which is known as florigen (Turck et al., 2008).

As mentioned previously, Cannabis is particularly sensitive to alterations in the photoperiod and as such the photoperiodic pathway of Arabidopsis warrants some more detailed discussion. The photoperiodic flowering pathway depends on cross-talk between light perception and the circadian clock, which coordinate to control the expression of the main integrator $F T$ (Cao et al., 2017). The first step in the photoperiodic pathway is the perception of light by the photoreceptors (phytochromes and cryptochromes). Phytochromes exist in inactive (Pr) and active (Pfr) forms. Pr is synthesised in the dark, and upon red-light perception is activated to Pfr which translocates to the nucleus. Pfr can interact with transcription factors and induce large-scale transcriptional alterations in response to light (Legris et al., 2019). Pfr then reverts to Pr by far-red light absorption or by light-independent thermal reversion (Klose et al., 2020). Phytochromes have a myriad of roles in regulating plant development and several phytochromes exist in angiosperms. The Brassicaceae possess five phytochromes: phyA to phyE. In Arabidopsis phyA and phyB are functionally the most important (Legris et al., 2019).

The photoreceptors subsequently transmit signals to the central node of the photoperiodic pathway: the GIGANTEA-CONSTANS-FT(GI-CO-FT ) signalling cascade. Briefly, the action of the GI-CO-FT module in Arabidopsis is as follows: the active Pfr form of phyA promotes the stability of the nuclear transcription factor CONSTANS (CO) which activates transcription of FT(Putterill et al., 1995; Samach et al., 2000). From the FT locus, florigen is produced, a small mobile protein which travels via the phloem from the leaves to the shoot apical meristem to induce the transition from vegetative to reproductive growth (Corbesier et al., 2007). The circadian clock gene GIGANTEA (GI) allows the degradation of transcriptional repressors that repress the expression of $C O$ thus indirectly promoting $F T$ (Sawa et al., 2007). The MADS-box transcription factor gene $S O C 1$ is indirectly upregulated by $C O$ via florigen. SOC1, in turn, activates the floral meristem identity gene $L E A F Y$, thus promoting flowering (Lee et al., 2008; Yoo et al., 2016).

Importantly, $S O C 1$ is a major floral integrator of different flowering pathways in Arabidopsis. For example, another MADS-box gene, FLOWERING LOCUS C (FLC), which is involved in the vernalization pathway directly binds to the $S O C 1$ promoter, and blocks $S O C 1$ transcriptional activation by CO (Hepworth et al., 2002). FLC also represses $F T$ transcription in the leaves and blocks florigen transport thus inhibiting flowering (Searle et al., 2006).

GI, CO and FT seem to be conserved in flowering pathways in many crops, such as wheat, barley, grapevine, pea, tomato, onion and cucurbits (Watanabe et al., 2011 and references therein). Thus, these genes are promising candidates for flowering time control in Cannabis. However, the gene functions and mechanisms controlling the flowering pathways may differ between species, and thus must be elucidated in Cannabis.

Several of these key regulators of flowering time have been demonstrated to have pleiotropic effects on agronomically valuable characteristics, further emphasising the importance of elucidating the role of these regulators in crop species (Blümel et al., 2015).

Given that Cannabis is a eudicot, short-day plant, the commonly used models- the long-day Arabidopsis or the monocot rice - may not be the most applicable for comparative analysis. Glycine max(soybean) is a short-day crop that belongs to the Fabales and is, therefore, more closely related to Cannabis (Rosales) than rice (Poales) or Arabidopsis (Brassicales) (Figure 3). Flowering time control in soybean is well studied and may provide important clues about how flowering is regulated in Cannabis.

In soybean, the $E$ genes and the JUVENILE $(J)$ gene are involved in flowering time control (Figure 8) (Copley et al., 2018 and references therein). $J$ is also named GmELF3, it is orthologous to Arabidopsis EARLY FLOWERING3 (ELF3), which is an important part of the circadian clock (Lu et al., 2017). Individuals that carry loss-of-function alleles for $E 1$ to $E$ / exhibit photoperiod insensitive flowering as higher transcript levels of the FT genes are present (Figure 8) (Xu et al., 2013).E1 is a legume specific transcription factor and the remaining genes are orthologous to those involved in flowering time control in Arabidopsis: 
E2 (also named GmGIa) is an ortholog of GI, and E3 (GmPhyA3) and E4(GmPhyA2) are orthologous to PhyA.

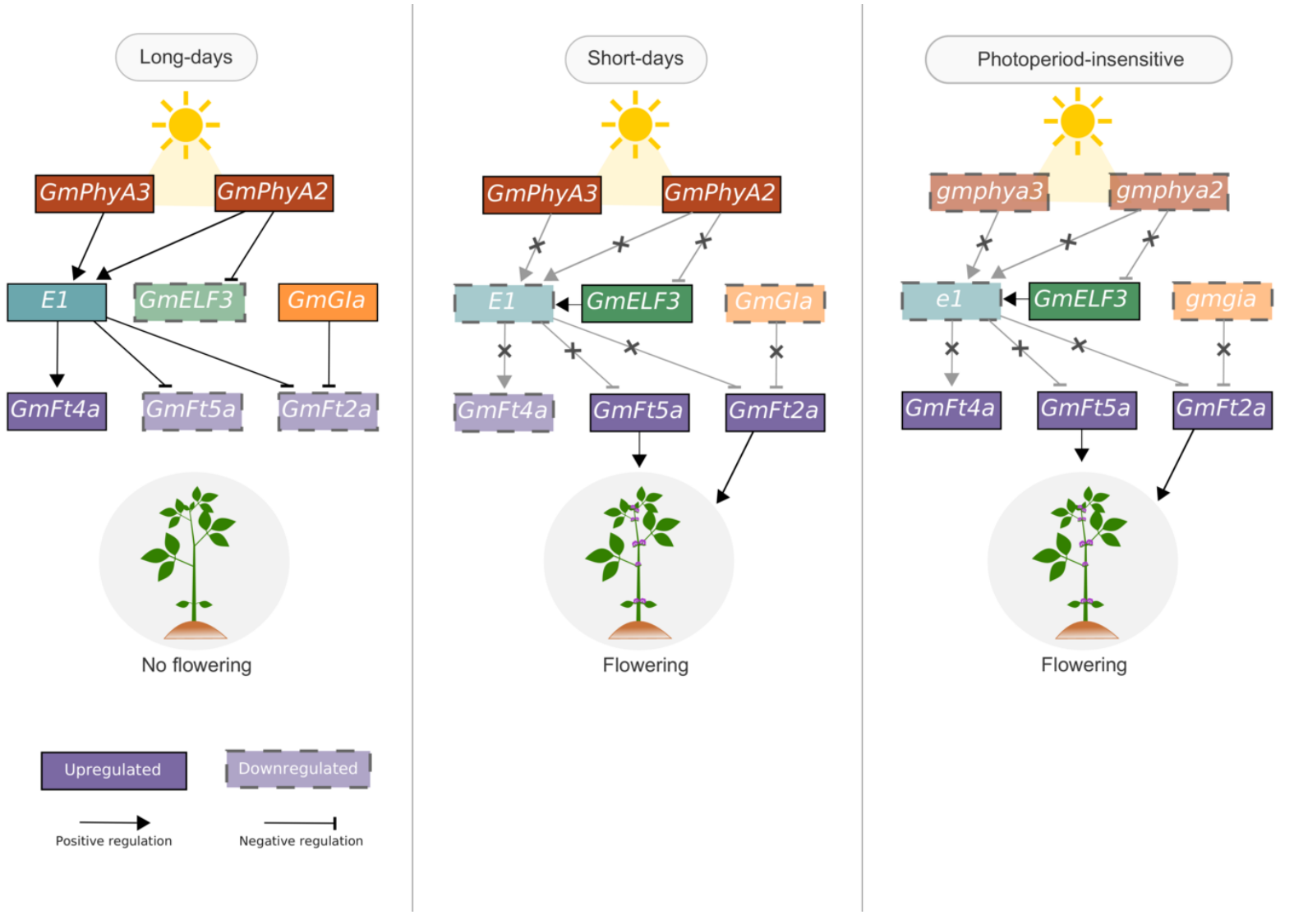

Figure 8: An overview of the working model for the photoperiodic flowering time pathway in soybean. Parallel representations of the network under long-day conditions and short-day conditions are shown as well as the genes that when mutated infer photoperiod-insensitive flowering. Under longday conditions, Phytochrome A homologs GmPHYA3 and GmPHYA2 promote E1 expression and inhibit GmELF3 expression. E1 up-regulates GmFT4a (a change-of-function $F T$ that suppresses flowering) and down-regulates GmFT2 $a$ and GmFT5a, all of which are FT homologs (Nan et al., 2014; Samanfar et al., 2017; Xia et al., 2012; Zhai et al., 2014). GmGIa is a GI homolog which inhibits GmFT2a (but interestingly not $G m F T 5 a$ ) thus delaying flowering under long-day conditions (Watanabe et al., 2011). Under short-day conditions, GmELF3 is expressed. GmELF3 represses $E 1$ by physically associating with the $E 1$ promoter. This leads to the release of the E1 suppression of the GmFT genes, thus promoting flowering under short days (Lu et al., 2017; Xia et al., 2012). Natural variation in the GmFT gene family is at least partially responsible for flowering time variation in soybean, with several polymorphic sites significantly associated with flowering time variation (Jiang et al., 2019). Soybean plants which carry loss-of-function alleles for E1, GmGIa, GmPHYA3 and GmPHYA2 exhibit photoperiod insensitive flowering as higher transcript levels of the $F T$ genes are present (Xu et al., 2013). Thus, these genes may represent strong candidates for elucidating photoperiod-insensitivity in Cannabis.

Natural variation in the $E$ and $J$ genes are responsible for the adaptability of soybean to cultivation at various latitudes, including the tropics, with several polymorphic sites significantly associated with flowering 
time (Jiang et al., 2019; Lu et al., 2017). Thus, these genes may represent strong candidates for elucidating the natural variation that exists for photoperiod sensitivity in Cannabis.

However, though candidate gene searches can be informative, it is worth considering that gene functions may not be similarly conserved across species. As such gene mapping approaches (genome-wide association studies, quantitative trait loci mapping) and functional analyses are still required to elucidate the flowering time network in Cannabis. Furthermore, as a wind-pollinated, dioecious species Cannabis is primarily outcrossing (Salentijn et al., 2015). In self-pollinating species such as rice, Sorghum and Arabidopsis, variation in flowering time is controlled by a few large-effect loci (Gage et al., 2020). However, in the model outcrossing species maize, several loci all contribute a small amount to phenotypes of complex traits such as flowering time (Buckler et al., 2009). It remains to be seen how complex traits will be determined in Cannabis, but taking inspiration from the successful elucidation of complex traits in maize may motivate the formation of sophisticated multi-parental mapping populations such as Nested Association Mapping (NAM) or Multiparent Advanced Generation InterCross (MAGIC) populations (Gage et al., 2020).

\section{The long read: Cannabis genetics and genomics}

While the medicinal, agricultural, and industrial applications of Cannabis are vast, Cannabis genomics has lagged in relation to that of other crops. In recent years, however, as legal restrictions have eased and with the advent of third-generation sequencing, the field has picked up significantly. With this, there is now a wealth of novel data readily available for analysis. Here, the currently available genomics and transcriptomics data will be reviewed.

The Cannabis genome is diploid $(2 \mathrm{n}=20)$, consisting of 9 autosomes and a pair of heteromorphic sex chromosomes ( $\mathrm{X}$ and $\mathrm{Y}$ ). The haploid genome size is predicted to be $843 \mathrm{Mb}$ and $818 \mathrm{Mb}$ for male (XY) and female (XX) plants respectively, with the larger Y chromosome accounting for the sex-specific difference in genome size (see also discussion above on sex determination) (Sakamoto et al., 1998). The Cannabis genome is not large relative to that of other crops like maize and wheat. However, it has been difficult to resolve due to its high heterozygosity and the abundance of repetitive DNA sequences. High heterozygosity levels have been retained in the genome, as Cannabis is dioecious and has not been subject to intense breeding (Lynch et al., 2016; Sawler et al., 2015). While this genetic diversity is desirable for selective breeding, it can complicate genome assembly. Highly variable alleles are often misassembled as segmental duplications, whereby both haplotypes are incorporated at distinct loci, inflating the genome assembly size (Claros et al., 2012; Michael and VanBuren, 2020). Additionally, it is estimated that repetitive sequences constitute $\sim 70 \%$ of the Cannabis genome (Gao et al., 2020; Laverty et al., 2019; Pisupati et al., 2018). Misassembly of multiple repeat elements onto one contig causes assembly collapse, reducing the genome assembly size (Claros et al., 2012; Michael and VanBuren, 2020). These features are particularly challenging when shortread sequencing is applied. While the first draft Cannabis genome, from the marijuana Purple Kush (PK) cultivar, was sequenced in 2011, the short-read sequencing of that time could not resolve repeat-rich, low complexity regions (van Bakel et al., 2011). This led to a very valuable yet incomplete genome assembly, consisting of $534 \mathrm{Mb}$ (van Bakel et al., 2011).

Third generation single molecule (or long-read) sequencing, such as Pacific Biosciences (PacBio) and Oxford Nanopore sequencing, can generate long reads which are capable of capturing the regions flanking repeat sequences and segmental duplications. Thus, long-read sequencing greatly facilitates the assembly process and has been revolutionary for plant genomics, enabling chromosome-level assemblies to be achieved (Jiao and Schneeberger, 2017; Michael and VanBuren, 2020). Recently, long-read sequencing, in parallel with genetic and physical mapping, has enabled four chromosome-level assemblies from the CBDRx, PK, Finola (FN), and a wild Cannabis (CR) line (Table 1) (Gao et al., 2020; Grassa et al., 2018; Laverty et al., 2019). The CBDRx genome (a female individual) was sequenced using Oxford Nanopore technology and has an assembly size of $876.148 \mathrm{Mb}$ (Figure 5a) (Grassa et al., 2018). In 2019, the first genome-wide annotation was made available for this Cannabis genome, making it the reference genome on the NCBI database (Jenkins and 
Orsburn, 2019). The PK and FN genomes were sequenced with PacBio single-molecule sequencing (Laverty et al., 2019). The PK (female) and FN (male) assembly sizes are 891.965 Mb and 1009.67 Mb respectively, both of which are significant improvements upon the original draft PK genome from 2011 (Table 1) (van Bakel et al., 2011; Laverty et al., 2019). The CR variety, which is derived from a wild Cannabis plant, was also sequenced with PacBio, achieving a genome assembly size of $812.525 \mathrm{Mb}$ (Gao et al., 2020). While linkage maps were generated to resolve the chromosomes for the CBDRx, PK and FN genomes (Grassa et al., 2018; Laverty et al., 2019), Hi-C data was used to create a physical map for CR, enabling the chromosomes to be assembled (Supplementary Table 1) (Gao et al., 2020).

Eight additional genomes have been assembled to varying levels of completeness (Table 1). Amongst these are the genome sequences of a father-mother-daughter trio from the Jamaican Lion (JL) cultivar, which was sequenced using PacBio (McKernan et al., 2020). The parental genome assemblies including gene annotation are available on the NCBI database, while all three genome assemblies are available on the Medicinal Genomics website (https://www.medicinalgenomics.com/jamaican-lion-data-release/). In addition to these three genomes, 40 genomes from a diverse range of cultivars were sequenced with Illumina short-read sequencing as part of the Medicinal Genomics 'Cannabis Pan-Genome Project' (McKernan et al., 2020). The whole-genome sequencing (WGS) data generated in this project are available on the NCBI sequence read archive (Supplementary Table 2). These genome sequences will be an invaluable resource for characterising the genetic basis behind the wide phenotypic diversity observed within Cannabis. Specifically, they will facilitate the development of a Cannabis pan-genome, where gene sets unique to specific cultivars could be defined. Such cultivar-specific genes are often representative of niche phenotypic adaptations that have evolved in response to specific environmental conditions (Montenegro et al., 2017; Tao et al., 2019). Cultivarspecific genes could be key targets for breeding, where new cultivars could be designed with desirable traits for specific production purposes (Tao et al., 2019).

There is also a wealth of additional genomics data available. This includes sequences of organellar genomes, of which there are seven mitochondrial and nine chloroplast genome assemblies available (Supplementary Table 3). The organellar genomes are particularly useful for resolving phylogenetic relationships. The rate of nucleotide substitution of mitochondrial coding sequences is lower than that of the nuclear and plastid genomes, making them useful molecular markers for resolving deep taxonomic relationships (Knoop, 2004; Wolfe et al., 1987). Despite this high intragenic sequence conservation, angiosperm mitochondria can exhibit high variation in genome organisation both within and between species (Cole et al., 2018; Davila et al., 2011; Palmer and Herbon, 1988). Perhaps taking a comparative genomics approach to investigate organisational variation within the mitochondrial genome between different Cannabis cultivars would be insightful for resolving relationships within the Cannabis genus. In contrast, the chloroplast genome is characterized by both stability in genome organisation and sequence conservation between species (Palmer and Herbon, 1988). Hence the chloroplast genome is often used to resolve phylogenies at the ordinal and familial taxonomic levels (Oh et al., 2016; Vergara et al., 2015; H. Zhang et al., 2018).

Furthermore, genotyping by sequencing (GBS), amplicon sequencing, bisulfite sequencing and Hi-C data are available for a multitude of different hemp as well as marijuana varieties (Supplementary Table 2). GBS is an efficient and cost-effective method to genotype a large number of samples, providing insight into the population structure and genetic diversity within a species (He et al., 2014). There have been at least three population-based studies that have generated GBS data for $\sim 400$ samples, representing both hemp and marijuana lines (Lynch et al., 2016; Sawler et al., 2015; Soorni et al., 2017). These studies find that hemp and marijuana often form distinct populations, not segregating based only on the $\mathrm{B}_{\mathrm{T}}$ and $\mathrm{B}_{\mathrm{D}}$ loci, but on a genome-wide level (Lynch et al., 2016; Sawler et al., 2015; Soorni et al., 2017). Bisulfite sequencing detects DNA methylation and is useful for understanding epigenetic gene regulation (Elhamamsy, 2016; Li et al., 2020). Two bisulfite sequencing datasets are available for analysis (McKernan et al., 2020; Niederhuth et al., 2016). Given that economically important traits like sex expression and flowering time are under strong environmental control, it will be interesting to explore to which extent those traits are epigenetically regulated. This may open the possibility of breeding 'climate smart' Cannabis plants, similarly to other crops where epigenetically regulated heat, drought or cold adaption are explored for crop improvement (Varotto 
et al., 2020).

Lastly, the 3D organisation of the genome within the nucleus can be mapped with Hi-C data (RodriguezGranados et al., 2016). One Hi-C dataset exists for the JL cultivar and is available on NCBI (Gao et al., 2020). Additional Hi-C datasets are available for the Jamaican Lion genomes through the Medicinal Genomics website (https://www.medicinalgenomics.com/jamaican-lion-data-release/). The 3D organization of the genome and its implications for gene regulation are currently being heavily investigated in plants (Santos et al., 2020). The available Cannabis Hi-C data are both useful for facilitating genome assembly as well as for understanding epigenetic regulation of gene expression (Burton et al., 2013; Lieberman-Aiden et al., 2009; Xie et al., 2015).

There have also been many studies that have focused on characterising the Cannabis transcriptomes (Supplementary Table 2). Perhaps most notably, in 2019, an extensive 'transcriptome atlas' was generated for Cannabis(Braich et al., 2019). This study involved RNA-sequencing of 71 samples taken from multiple tissues of the Cannbio-2 cultivar (CN2), at various developmental stages. This transcriptome data will be useful for the annotation of new genome assemblies, as well as for inferring gene functions based on spatiotemporal gene expression patterns. Other studies have characterised the transcriptome of hemp lines grown under salinity and drought stress (Gao et al., 2018; Liu et al., 2016), as well as during bast fibre development (Behr et al., 2016; Guerriero et al., 2017). Three further studies have focused on sequencing the transcriptome of glandular trichomes, with the aim of profiling the expression of genes involved in terpene and phytocannabinoid biosynthesis (Booth et al., 2020; Livingston et al., 2020; Zager et al., 2019). Furthermore, two recent studies have focused on identifying the sex chromosomes based on characterising the expression of sex-linked genes in male and female plants (McKernan et al., 2020; Prentout et al., 2020). The transcriptomes of the PK and FN cultivars sequenced in 2011 are also available (van Bakel et al., 2011).

While wide-spread illegalization of Cannabis has stunted genomics research in the past, it is clear that there have been major advances in this field in recent years. With chromosome-level genome assemblies now available, as well as genome-wide annotations and abundant transcriptome data, the resources for future research are plentiful.

\section{More than the sum of its parts: Medical applications of phyto- cannabinoids}

Cannabis plants represent a rich source of biologically active compounds, including more than 100 plantderived cannabinoids (phytocannabinoids) and more than 200 terpenoids (Russo, 2011). Thus far, research into the medicinal effects of Cannabis has largely focussed on phytocannabinoids. Among these, the most well-studied are the psychoactive THC, and the non-psychoactive CBD, though other phytocannabinoids such as CBG and CBC also show therapeutic potential (Russo, 2011) (see chapter 3 for details on phytocannabinoid synthesis and genetics).

\subsection{Cannabis metabolites}

Early investigations into the pharmacologic effects of THC led to the discovery of the human endogenous cannabinoid (endocannabinoid) system, which includes endogenous cannabinoid ligands, metabolic enzymes, and the two major cannabinoid receptors, CB1 and CB2 (Izzo et al., 2009). The modes of action of phytocannabinoids are complex, with individual compounds capable of acting at multiple molecular targets. THC modulates the activity of $\mathrm{CB} 1$ and $\mathrm{CB} 2$ but can also activate the transcription factor PPAR $r$ and the TRP ion channel TRPA1 (Izzo et al., 2009). CBD, meanwhile, has low affinity for CB1 and CB2, but can modulate the activity of various components of the endocannabinoid system. Furthermore, like THC, CBD can target PPAR $\gamma$ and TRPA1, as well as the G-protein coupled receptors GPR55 and GPR18, the TRP ion channels TRPV1, TRPV2 and TRPM8, and the serotonin receptor 5-HT $1 \mathrm{a}$ (Izzo et al., 2009). By 
modulating various signalling pathways involved in multiple diseases, phytocannabinoids have the potential to provide many therapeutic benefits.

The analgesic, antiemetic and anticonvulsant properties of phytocannabinoids are well-established (Whiting et al., 2015). Growing evidence suggests that Cannabismetabolites also produce anti-inflammatory, antidepressant, anxiolytic and anti-cancer effects (Atalay et al., 2019; Fraguas-Sánchez and Torres-Suárez, 2018; Poleszak et al., 2018; Śledziński et al., 2018). Synthetically produced cannabinoids can mimic the effects of plant-derived compounds, and many countries have approved synthetic cannabinoids for medicinal use. Dronabinol and nabilone, synthetic forms of THC, are approved for the treatment of chemotherapy-induced nausea and vomiting, and for appetite stimulation in AIDS-associated anorexia (Freeman et al., 2019). However, plant-derived medicines have also been developed, namely Epidiolex, a purified form of CBD for the treatment of severe forms of epilepsy, and Sativex, a Cannabis extract containing THC and CBD for the management of pain and spasticity in multiple sclerosis (Freeman et al., 2019).

Additionally, Cannabis contains many non-cannabinoid metabolites, including terpenoids, flavonoids, ligand amides and stilbenes (Pollastro et al., 2018b). Of these, the terpenoids have been most extensively studied, and display many therapeutic effects (Russo, 2011). Due to growing evidence that various Cannabis bioactive compounds act synergistically to produce therapeutic effects (Russo, 2011), a greater understanding of the pharmacological contributions of differentCannabis metabolites will be needed to develop the most effective Cannabis -based medicines.

\subsection{Cannabis synergy}

To date, research into Cannabis-based medicines has primarily focussed on single isolated cannabinoid compounds. However, some studies show that combinations of various Cannabis components display greater biological activity than single compounds, suggesting that whole plant extracts may be more effective than purified phytocannabinoids (Russo, 2011). The increased activity of whole Cannabis extracts may be due to the synergism between various cannabinoid and non-cannabinoid components, which has been termed 'the entourage effect' (Russo, 2011). Proposed mechanisms underlying the entourage effect in Cannabisinclude activation of multiple molecular targets, enhanced bio-availability or solubility of compounds, and neutralisation of adverse events (Wagner and Ulrich-Merzenich, 2009). Exploiting Cannabis synergy to develop new medicines based on whole-plant extracts may be beneficial for a range of pharmaceutical applications.

In epilepsy, the addition of non-THC, non-CBD Cannabismetabolites may enhance the anticonvulsant effects of existing treatments. In a mouse model of epilepsy, the content of minor phytocannabinoid compounds in a high-CBD plant extract treatment affected seizure incidence and survival rates, suggesting that specific combinations of phytocannabinoids may be more effective than single purified compounds (Berman et al., 2018). Furthermore, a meta-analysis of observational clinical studies on epilepsy treatment reported that CBD-rich plant extracts reduced seizure frequency in patients at doses lower than those used in clinical trials of Epidiolex. The CBD-rich plant extract also produced significantly fewer adverse effects, likely due to the lower dose required (Devinsky et al., 2017; Pamplona et al., 2018; Thiele et al., 2018).

The benefits of phytocannabinoids for pain management have been well-established. However, the analgesic effects of Cannabis may be enhanced by combining different Cannabis bioactive compounds. The analgesic and anti-inflammatory effects of CBD occur only within a very limited dose range, but this bell-shaped doseresponse was overcome when CBD was combined with a Cannabis extract (Gallily et al., 2015). Another study showed that a Cannabis extract high in THC provided no benefit for intractable cancer pain, while nabiximols, a whole extract $\mathrm{CBD} / \mathrm{THC}$ combination significantly reduced pain in cancer patients (Johnson et al., 2010).

Whole-plant extracts may also be preferable to single phytocannabinoids for the treatment of mood disorders. Several phytocannabinoids, including THC, CBD and CBC, appear to have antidepressant and/or antianxiety effects, possibly due to modulation of the endocannabinoid system and/or modulation of serotonin receptors (Crippa et al., 2011; Poleszak et al., 2018; Zanelati et al., 2010). Other non-cannabinoid Cannabis 
compounds, such as the terpenoid limonene, also exhibit antidepressant effects, and lemon oil, which contains high quantities of limonene displays anti-stress and anxiolytic properties (Komiya et al., 2006; Piccinelli et al., 2015; Russo, 2011). Plant extracts containing both cannabinoids and terpenoids may be the most effective Cannabis-based treatment option for psychopharmacological applications.

Synergistic effects are also potentially beneficial for cancer treatment. In breast cancer cell lines and animal models, a Cannabis extract produced enhanced anti-tumour effects compared to purified THC, possibly due to the presence of other cannabinoid compounds (Blasco-Benito et al., 2018). Another study reported that whole plant extracts reduced cancer cell survival and proliferation more effectively than pure THC, across a range of cancer cell types (Baram et al., 2019). Notably, one study found that cancer cells were killed most effectively when treated with phytocannabinoids and terpenoids at ratios similar to those found naturally in the plant (Namdar et al., 2019).

The above findings suggest that, for the treatment of various medical conditions, producing a range of Cannabis varieties, or chemotypes, with varying phytochemical contents may constitute a more effective approach than developing new synthetic cannabinoid-based medicines. A greater understanding of the synergistic activities of different phytocannabinoids, terpenoids and other Cannabiscomponents is needed to identify the most effective combinations for various pharmaceutical applications.

\subsection{Cannabis breeding for medicine}

Cannabis sativa is a versatile multi-purpose crop which requires a simple, low-input cultivation technique, adapts to various ecological conditions, produces sustainable products, and provides raw material for a wide range of applications, including medicine (Amaducci et al., 2015). Research into the synergistic pharmacological effects of Cannabis metabolites suggests that ratios of phytocannabinoids, terpenoids and other Cannabis metabolites influence a plant's therapeutic potential.

More research is needed to determine the influence of environmental and genetic factors on the phytochemical profile of the Cannabisplant. Existing studies show that total phytocannabinoid yields are related to environmental conditions. Phytocannabinoid and terpene levels are affected by factors such as the humidity, rainfall and temperature of the growth environment (Meier and Mediavilla, 1998; Murari et al., 1983; Pavlovic et al., 2019). However, the relative ratios of the different Cannabis metabolites are dependent on the genotype (see Chapter 3) (Janatová et al., 2018; de Meijer et al., 2003; Toonen et al., 2006; Vanhove et al., 2011). Identifying the environmental and genetic factors that influence phytochemical production by Cannabis could aid in the development of new Cannabis cultivars with tailored ratios of various metabolites.

The therapeutic effects of a given plant reflects the proportions of the various pharmacologically active components. The development of Cannabis chemotypes containing high levels of specific phytocannabinoids can be achieved through breeding. De Meijer et al . produced Cannabis chemotypes high in specific single phytocannabinoids, including THC, CBD, CBG and CBC (de Meijer et al., 2009a, 2003; de Meijer and Hammond, 2005). Cannabinoid-free chemotypes were also developed, which could aid investigation into the contributions of non-cannabinoid bio-actives, such as terpenoids, to the pharmacological effects of Cannabis (de Meijer et al., 2009b; Russo, 2011). The development of these chemotypes through conventional breeding demonstrates the high diversity of the Cannabis genome, which may obviate the need for genetic engineering of Cannabis(Russo, 2019).

Understanding the interactions between different Cannabisbio-actives remains one of the key challenges to harnessing the full medicinal potential of the Cannabis plant. Research into Cannabis-based medicines highlights the importance of various Cannabis metabolites in producing therapeutic effects. Further research is needed to elucidate the mechanisms underlying the entourage effect observed with whole Cannabis extracts, and to assess the contributions of various Cannabis metabolites to determine the most effective combinations for various pharmaceutical applications. Applying our knowledge of the entourage effect in Cannabis to the development of tailored chemotypes has the potential to provide improved Cannabis-based therapies for various medical conditions, which could benefit many patients. 


\section{Hemp for houses: Cannabis as building material}

Many hemp varieties of Cannabis are fibre crops with multiple inherent qualities as building material. The high tensile strength of hemp fibres, traditionally exploited in rope and fabric applications, also enables mechanical advantages for building construction applications. Additionally, shiv particles of the woody-core are a biobased alternative to mineral aggregates for low-impact concrete. Therefore both the plant fibres and the shiv particles are suitable for developing biobased, environmentally friendly building materials that have been shown to have inherent thermal, hygrothermal and acoustic characteristics (Kinnane et al., 2016; Reilly et al., 2019; Shea et al., 2012).

Building products that integrate hemp are many, but reductively may be grouped into two general categories, hemp concrete and hemp insulation blankets: Hemp concrete are those mixed with a binder to form a porous concrete composite with thermal insulation qualities. Hemp insulation blankets are thermo-formed without an added binder to create a low density, blanket type product. These respectively encompass shiv and fibres, and are distinguished by their dry densities; typically in the range $390-670 \mathrm{~kg} / \mathrm{m}^{3}$ in the case of the hempconcrete (Collet-Foucault et al., 2004), and about $38 \mathrm{~kg} / \mathrm{m}^{3}$ for the hemp-insulation ('Technichanvre', 2017). The concretes have a wide density range as they are typically bespoke and include varying levels of binders, often lime, but also cement. As noted, both products are recognised for their good thermal properties. The hempcretes have a low thermal conductivity $\left(\lambda=0.12 \mathrm{~W} /\left(\mathrm{m}^{*} \mathrm{~K}\right)\right.$ ) (Walker and Pavía, 2014) relative to other standard concretes $\left(\lambda=1-2 \mathrm{~W} /\left(\mathrm{m}^{*} \mathrm{~K}\right)\right)$. However, their conductivity is higher than the former insulation wool blanket product $\left(\lambda=0.04 \mathrm{~W} /\left(\mathrm{m}^{*} \mathrm{~K}\right)\right)$ (Collet-Foucault et al., 2004), which contains up to $90 \%$ hemp fibre, are formed in panels or rolls and used for roof, attic and wall insulation.

Hempcrete, in contrast, is a composite material. It is commonly mixed in a ratio by weight of 1:2:3 of hemp: binder: water. In contrast to standard concrete, hempcrete has relatively low mechanical strength. It is therefore typically cast around a load-bearing timber structure. The wet mix is poured between temporary shuttering, and the hempcrete is tamped down to compact it and form the wall. The thickness of these walls typically ranges from $300-600 \mathrm{~mm}$, to ensure structural stability and to meet thermal requirements. These dimensions limit the widespread applicability of hempcrete, particularly in urban infill sites. However, new innovative products are enabling its wider applicability. Increasingly, precast hempcrete blocks are appearing on the market. Exhibiting higher densities, certain of these have load-bearing capability and they generally enable time and labour efficiencies on site. They are also popular for renovation and retrofit projects, primarily because hempcrete is characterised by an open pore structure which allows for the transmission of moisture. Moisture is often present in the walls of old buildings, and breathable insulation allows this water escape, instead of trapping it as do modern-day synthetic insulations which can lead to mould growth, structural and air quality issues.

These are just some example of advantages of biobased materials. However, the construction industry and the agricultural industry diverged during the modern post-war age of development. Synthetic products were developed to meet high demand with a price point that enabled use, and waste, of products during phased redevelopment (Kinnane, 2020). Today synthetic polymer and mineral wool products continue to command almost full market share of the insulation industry, and that demand is increasing as we aim to reduce the operational energy of buildings. Hemp, and biobased materials more generally, remain niche products. Today hemp insulation products are almost twice the price of the mass produced alternatives (Carus et al., 2013), even though plant fibres have a lower cost of processing than synthetic fibres.

However, the building sector, and its considerable environmental impact, is increasingly in focus, and the environmental benefits of biobased materials are giving them greater traction. Hemp, with its fast-growth cycle and multi-purpose advantages is increasingly proposed as a low-impact design solution. Although specific quantification of carbon sequestration remains challenging (Reilly and Kinnane, 2017), authors report levels of between 1.5-2.1 $\mathrm{kgCO}_{2}$ per $\mathrm{kg}$ of plant grown and values of energy for production of $0.085-0.19 \mathrm{kgCO}_{2}$ per kg of hemp shiv (see Sáez-Pérez et al., 2020 for review). It should be noted however that although hemp exhibits carbon positive credentials, the embodied carbon of any hempcrete is high due to the high quantity 
of binders often used, and this is often underestimated by proponents of the material.

\section{Also a medicine for the environment? Sustainability aspects of Cannabis farming}

Considering global warming and consequential efforts to divest from fossil fuels, bioenergy crops and biofuels are gaining increasing interest (Rogelj et al., 2018). Cannabis is a high-yielding, annual crop, and has muchuntapped potential for contributing to carbon sequestration efforts (Finnan and Styles, 2013). Besides storing carbon in building materials, alternative uses for this crop exist, and as such, there is a high potential for carbon to be stored both short- and long-term in bioenergy, textiles, and paper (Figure 1, Figure 9). Further contributing to the environmental connotations of this species, hemp has been employed in phytoremediation efforts to restore land implicated by heavy metal contaminants (Citterio et al., 2003). Hemp leaves and seeds also provide the basis for human consumption (Figure 9).

Hemp is considerably more efficient (high annual yields with low agrochemical/fertilizer input) than the traditional annual bioenergy crops (sugar beet and oilseed rape) and possesses similar greenhouse gas mitigation potential to the perennial bioenergy crops Miscanthus and willow (Finnan and Styles, 2013). Annual bioenergy crops like hemp can be appealing options for farmers to diversify and explore the bioenergy market without the demands of perennials, namely high establishment costs and long-term commitment (15-20 years) of their land to bioenergy (Finnan and Burke, 2013; Finnan and Styles, 2013).

Hemp biomass has good combustion properties and could be used to generate either heat or electricity (Finnan and Styles, 2013). There are multiple biofuel options: Biogas, solid fuel briquettes, bales, and bioethanol (Kraszkiewicz et al., 2019; Prade et al., 2012, 2011).

As hemp is an annual crop it can be readily integrated into crop rotation cycles, thus not competing with food supplies and can therefore contribute towards sustainable cropping systems (Finnan and Styles, 2013). Moreover, hemp has been reported to improve yields of crops subsequently grown thus complementing food production. Winter wheat planted after hemp had 10-20\% yield increases (Bócsa and Karus, 1998), with similar observations recorded for soybean and alfalfa (Adesina et al., 2020). A low input crop, hemp can produce high yields similar to switchgrass and sorghum but with lower nutrient and pesticide requirements (Das et al., 2017). Hemp offers the combined potential of an effective break crop and an efficient energy crop, thus generating income while promoting productivity. Break crops like hemp can be used to disrupt pest cycles and the ability of hemp to tolerate high planting densities suppresses weed growth, thus pesticide and herbicide requirements of subsequently, cultivated crops are reduced (Bhattarai and Midmore, 2014). The hemp root system promotes soil health, as the large taproots penetrate deep into the soil facilitating aeration, but simultaneously forms soil aggregates to prevent soil erosion (Amaducci et al., 2008). Model analysis comparing the relationship between leaf nitrogen status and photosynthesis rate in hemp, cotton and kenaf revealed hemp to have a high photosynthetic capacity, even at low nitrogen levels (Tang et al., 2017). This provides an additional line of evidence that hemp may fulfil a future niche as a sustainable bioenergy crop that can be cultivated over a wide range of climatic and agronomic conditions. 

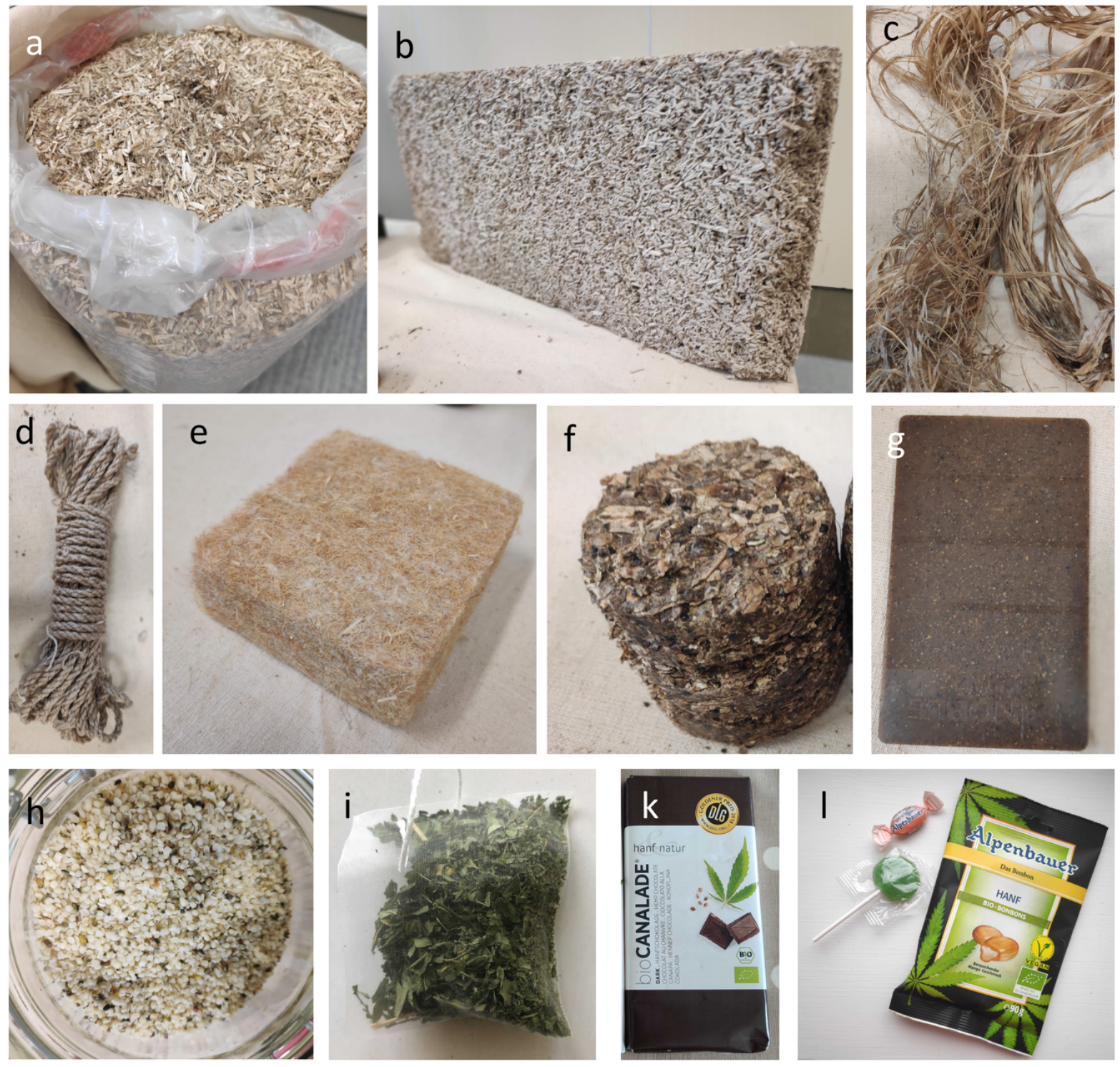

Figure 9: Products made from hemp. Non-THC Cannabis (hemp) can be processed into a multitude of different products. Hemp husk of fibre varieties (a) can be processed into hempcrete (b), while hemp fibre (c) can be made into rope (d) or insulation material (e). Remains of the plant can be pressed into hemp pellets for heating (f). Composite hemp-plastic material for 3D printing $(\mathrm{g})$ is more sustainable than regular printing plastic. Hemp seeds can be dehulled into hemp hearts for human consumption (h). A variety of hemp products like tea (i), as well as chocolate (k) and candy (l) are also available. (i) courtesy of Christine Schilling.

\section{Future prospects: Phytocannabinoids without Cannabis: In vi- tro synthesis using cell cultures}

Phytocannabinoids have high potential for medical but also recreational use and therefore their production and extraction are of high commercial interest. However, plant breeding and cultivation come with their 
own challenges and phytocannabinoid yield and profiles can highly depend on environmental factors. Cell cultures methods are a powerful tool for the production of high-quality plant material in a manner that is time efficient, seasonally independent, and which can satisfy good manufacturing practice guidelines (Tekoah et al., 2015). This technology has attracted a lot of attention as it can allow the harvesting of high value products produced within cells in suspension or secreted into their surrounding medium (Weathers et al., 2010). Improvement of culture growth kinetics and product yield can be achieved via medium optimisation (Holland et al. , 2010; Ullischet al. , 2012; Vasilev et al. , 2013) and by selecting for high-producing cell populations using techniques such as fluorescent marker-based cell sorting (Kirchhoff et al. , 2012). Cell lines optimised in these ways can subsequently be cryopreserved to ensure consistent production going forward (Ogawa et al. , 2012).

Secondary metabolites, including pharmacologically valuable compounds such as paclitaxel and scopolamine or transgenic proteins to be used as vaccines, antibodies, immunomodulators and other therapeutics are already produced in cell suspension cultures on a commercial scale (Mountford, 2010; Paul et al., 2015). Hence, this might be a promising avenue to produce cannabinoids as well (Figure 10).

Of the various types of plant cell culture available, cell suspension cultures are the most commonly used due to their scalability and relatively rapid growth rates (Santos et al., 2016). The use of cell suspension cultures involves growing dedifferentiated plant cells in liquid medium supplemented with hormones to induce culture proliferation (Mustafa et al., 2011). However, the use of suspension cultures for these purposes faces several barriers to successful execution. Firstly, due to their genetic instability, cultures can often lose their ability to produce valuable compounds over time, while low productivity rates sometimes require large volumes of biomass to be grown, therefore increasing costs relative to field-grown plants (Moon et al., 2020; Weathers et al., 2010). Cell suspension cultures tend to form heterogeneous cell clusters rather than proliferating as single cells in culture, leading to increased difficulty of use and inconsistent growth kinetics and product yield. In addition, the scale-up of cell suspension cultures from laboratory to commercial production scale is often associated with a decline in cell productivity (James and Lee, 2006).

In relation to Cannabis, the use of in vitro bioprocessing techniques has the potential to allow the synthesis of high yields of cannabinoids in a manner that satisfies good manufacturing practice guidelines and guarantees a high-quality product. Metabolic engineering offers the possibility of developing plant or microbial cell lines which exclusively produce a desired cannabinoid, thus circumventing the high costs associated with purifying a desired product during downstream processing. However, achieving these aims poses a number of challenges to which researchers must still find answers.

A study described by Pacifico et al., (2008) assessed the cannabinoid content of callus cultures (which are metabolically identical to and often constitute the starting material for suspension cultures) derived from five different Cannabis varieties. The calli did not show any detectable levels of phytocannabinoids at any time during culture, irrespective of the presence or absence of hormones or the phytocannabinoid content of the original plants from which the cultures were derived. As such, cell suspension cultures are unlikely to be an effective biofactory for the production of cannabinoids without some form of intervention.

One method which may overcome the lack of phytocannabinoid production in Cannabis suspension cultures is the use of elicitors. These are compounds or a mixture thereof which can be added to the culture medium to stimulate the transient production of a desired secondary metabolite. Elicitors can be either biotic (animal, plant or microbial extracts) or abiotic (metal ions, organic compounds or electric current) and have been used previously with varying degrees of success (Weathers et al., 2010 and references therein). However, since many elicitors are either toxic or stress-inducing, their addition to a suspension culture often leads to a reduction in the vitality of the culture and can even be fatal. Such an approach was used by Flores-Sanchez et al. (2009) in order to try to stimulate phytocannabinoid production in suspension cultures of Cannabis. However, no detectable levels of phytocannabinoids were found in response to any of the treatments used, which included a range of biotic and abiotic stimuli. As such, the search for an elicitor which can induce phytocannabinoid production remains ongoing. 


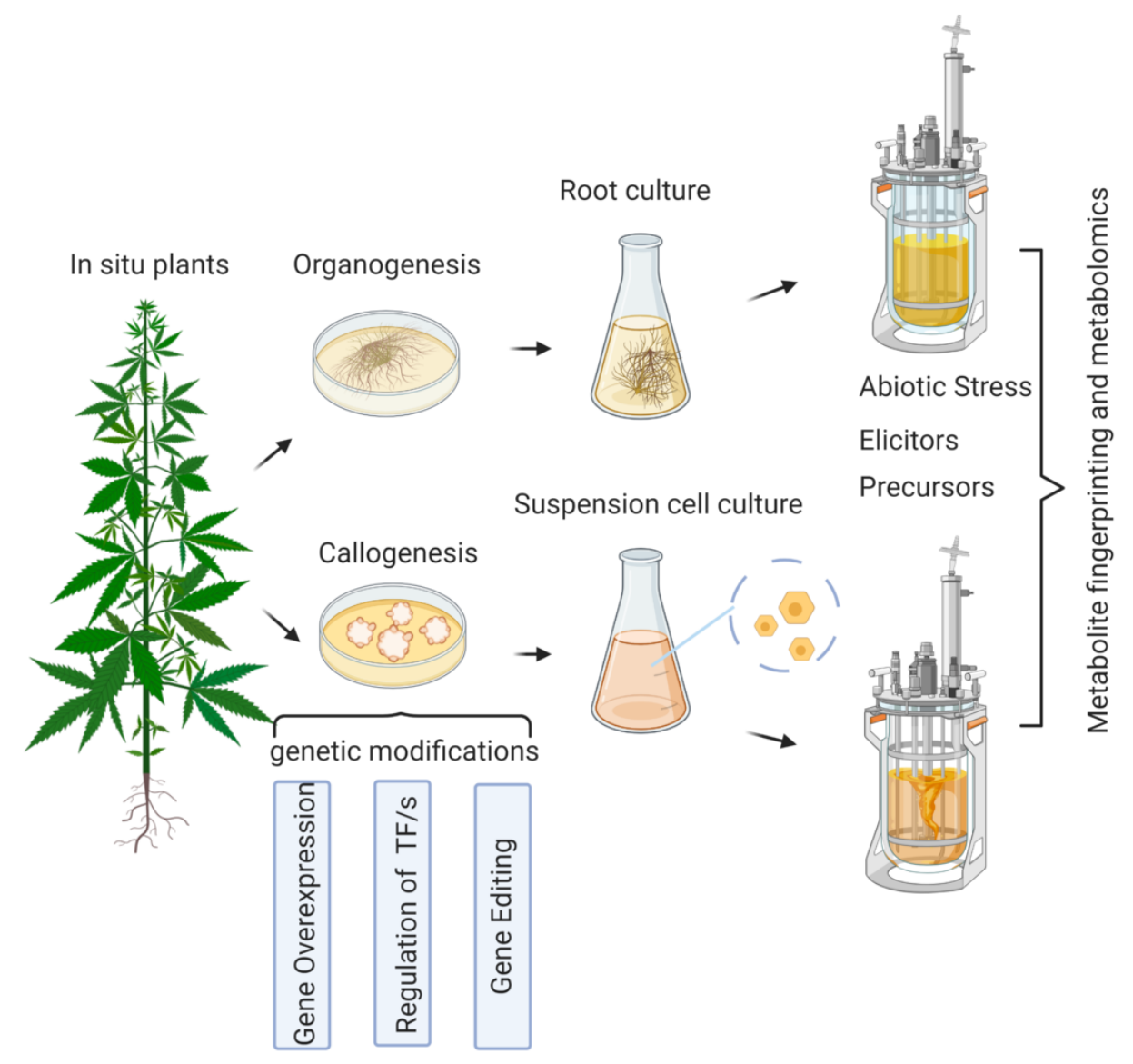

Figure 10: Developments in plant cell and tissue culture methodologies allow the efficient production of metabolites and recombinant proteins at commercial scales. Cell suspension cultures and hairy root cultures are the most commonly used techniques, with protocols described for both in Cannabis . However, efficient, large-scale production of phytocannabinoids in vitro has yet to be developed. Image was made using Biorender (www.biorender.com).

One other point worth noting is that phytocannabinoids are known to be toxic to plant cells when they accumulate at high enough concentrations, which is why Cannabis plants use trichomes to compartmentalise these compounds into storage cavities outside the plant. THCA and its precursor molecule CBGA are highly toxic to both Cannabis and tobacco cell suspension cultures, inducing $100 \%$ apoptotic-like programmed cell death after 24 hours in culture at a concentration of $50 \mu \mathrm{M}$ (Sirikantaramas et al., 2005). This negative feedback phenomenon is observed in many plant species which produce secondary metabolites and is the reason why many metabolites are sequestered in specialised structures.

However, the development of cell culture methods to avoid cell toxicity is one area where researchers have relative success. Strategies such as two-phase cultures have been shown to enhance the production of secondary metabolites in a range of species (Malik et al., 2013). In these systems, an aqueous phase is used to support cell growth while a non-aqueous phase, typically consisting of a solvent or resin, is employed to act as a sink for the accumulation of the desired product and in some cases facilitates its subsequent 
extraction. Two-phase systems have been shown to greatly increase metabolite yield in both plant cell suspension and hairy root culture cultures (Chiang and Abdullah, 2007; Malik et al., 2013; Rudrappa et al., 2004; Sykłowska-Baranek et al., 2019; Wu and Lin, 2003), although to the best of our knowledge such an approach has not yet been attempted in Cannabis.

Hairy root cultures are generated by the infection of plant tissues with Agrobacterium rhizogenes, a species which can modify the plant's genome by introducing a segment of DNA known as T-DNA which codes for a number of genes affecting the production and regulation of plant hormones (Ono and Tian, 2011). This results in the development of extensive root networks which can be easily cultured in vitro, are genetically identical to the mother organ from which they were derived and can also produce the same phytochemicals. Like cell suspension cultures, hairy root cultures have already attracted attention as a means of producing secondary metabolites such as flavonoids (Gai et al., 2015), isoflavonoids (Jiao et al., 2014), artemisinin (Patra and Srivastava, 2014) and lignans (Wawrosch et al., 2014), albeit less commonly than cell suspension cultures due to their increased difficulty of use.

THCA has previously been produced from tobacco hairy root cultures which were transformed to express the enzyme responsible for its production, THCA synthase, under the transcriptional control of the cauliflower mosaic virus 35S promoter (Sirikantaramas et al., 2004). When these hairy roots were cultured in liquid medium supplemented with CBGA, the precursor molecule to THCA, $8.2 \%$ of this CBGA was converted to THCA after two days of culture, approximately half of which was then secreted into the surrounding medium, thus demonstrating that CBGA uptake and THCA release from these transgenic roots was possible in vitro, albeit at low levels.

In Cannabis, a protocol for the production of hairy root cultures has already been described which shows that cultures are best established from the hypocotyl of intact seedlings by piercing the epidermis with a syringe and inoculating with $A$. rhizogenes(Wahby et al., 2013). Five varieties of Cannabis (three hemp-type and two marijuana drug-type) were used and all were shown to be responsive to infection by A. rhizogenes, although with varying morphological responses. Similarly, all eight $A$. rhizogenes strains used could induce a hairy root morphology, albeit with varying degrees of frequency (43-98\%, depending on the strain).

An alternative approach described by Farag and Kayser (2015) outlines how hairy root cultures can be developed from Cannabis callus cultures without the use of A. rhizogenes by growing them in B5 medium supplemented with $4 \mathrm{mg} / \mathrm{ml}$ of the auxin NAA. Under these conditions, cannabinoid contents peak at 1.04 $\mu \mathrm{g} / \mathrm{g}$ dry weight for THCA, $1.63 \mu \mathrm{g} / \mathrm{g}$ dry weight for CBGA and $1.67 \mu \mathrm{g} / \mathrm{g}$ dry weight for CBDA after 28 days of culture. These low yields highlight the fact that while phytocannabinoids can be produced from hairy root cultures, significant improvements in yield will need to be achieved before this methodology is commercially viable for phytocannabinoid production.

Recent studies have attempted to demonstrate the production of cannabinoids in non-native hosts, with a particular focus on yeast due to the relative ease with which metabolic engineering can be achieved in this model organism. A landmark paper by Luo et al. (2019) described the complete biosynthesis of CBGA, THCA and CBDA and several unnatural analogues in yeast via engineering of the native mevalonate pathway and the introduction of a heterologous hexanoyl-CoA biosynthetic pathway as well as the Cannabis genes responsible for the biosynthesis of complete cannabinoids. However, the cannabinoid yields achieved from this system were found to be approximately 100-fold less than those produced by Cannabis plants. As such, the efficient production of cannabinoids in either plant or microbial cell culture remains a work in progress.

\section{Conclusions: Stay tuned, there is more to come!}

The recent progress in Cannabis research has been remarkable, and has revealed exciting challenges ahead: The evolution and genetic diversity of phytocannabinoid synthases has proven to be a complex field of research, as is the genetic and environmental control of sex determination and flowering time. The increasing availability of genomic resources will undoubtedly facilitate progress in all those areas, but we predict that 
experimental analyses, including detailed morphological, molecular genetics and phenotyping studies will be equally important to understand the developmental and physiological intricacies of Cannabis.

Unusual in that it is a multipurpose crop, the full sustainability potential of Cannabis can only be fulfilled if it is used as such. Thus, one major challenge will be to design crop ideotypes that harmonise traits of medicinal relevance with those important for carbon sequestration. This will not be an easy task, as the genetic control of different traits is currently unclear. However, the production of e.g. large fibre varieties that do nevertheless develop a dense inflorescence with high CBD content seems not too farfetched. Even if those hypothetical cultivars may not be able to provide the high yields of specialized CBD cultivars they may provide farmers focusing on fibre production with a second source of income.

In summary, the genetic and morphological diversity of Cannabis is a treasure trove that we are only beginning to explore. It is important that we capitalise on this treasure to construct a multipurpose swiss knife, and not a series of highly specialised tools.

\section{Acknowledgements}

SS is grateful for support by a Postdoctoral Scholarship of the Irish Research Council and Greenlights Medicines under the Enterprise Partnership Scheme (Grant No.: EPSPD/2019/220). CAD is supported by an Irish Research Council-Environmental Protection Agency Government of Ireland Postgraduate Scholarship (Grant No: GOIPG/2019/1987). JS is supported by a Chinese Research Council Postgraduate Scholarship (Grant No: CSC no. 201908300031). EOR and ASP are supported by the Irish Research Council and Greenlight Medicines via an enterprise partnership scheme (Grant no.: EPSPG/2017/376).

RM and SS are grateful to Beate and Peter Melzer, Christine Schilling, and Andreas Schmidt for childcare and general support during the pandemic, and to Jonathan and Theodor Schilling for their patience during those unusual times. This substantially helped with writing this manuscript.

All authors are members of HempHub, a strategic initiative of the UCD Earth Institute, and are grateful for general support through this initiative.

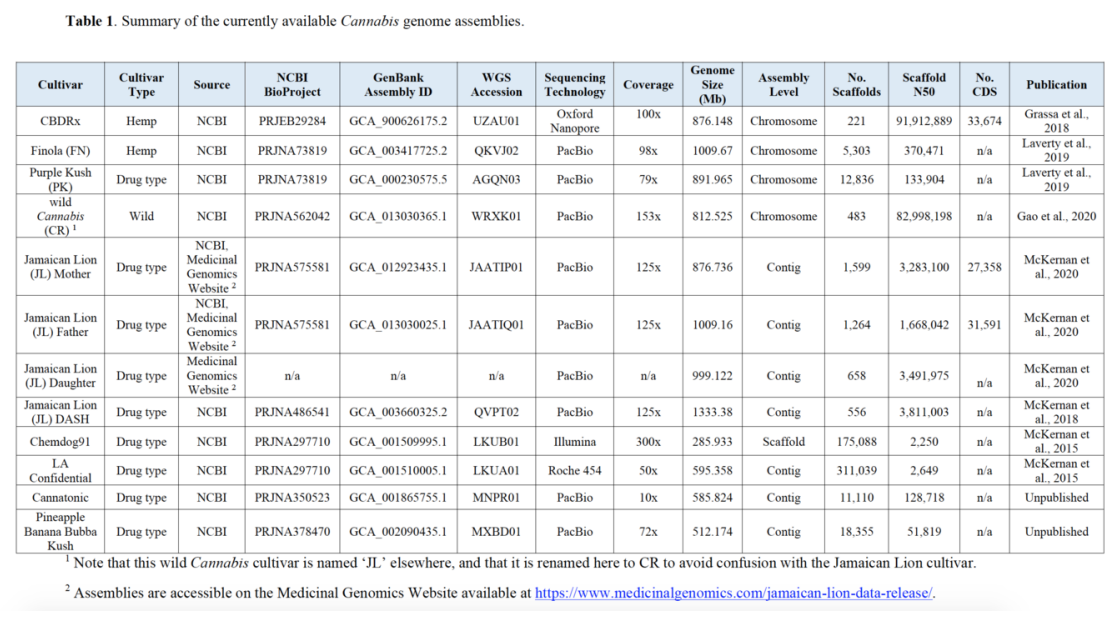

Figure 11: Table 1 - Excel and PDF attached

\section{Bibliography}

Adesina, I., Bhowmik, A., Sharma, H., and Shahbazi, A. (2020) A review on the current state of knowledge of growing conditions, agronomic soil health practices and utilities of hemp in the United States. Agriculture. 
10.

Ainsworth, C. (2000) Boys and Girls Come Out to Play: The Molecular Biology of Dioecious Plants. Ann Bot. 86: 211-221.

Aizpurua-Olaizola, O., Soydaner, U., Öztürk, E., Schibano, D., Simsir, Y., Navarro, P., et al. (2016) Evolution of the Cannabinoid and Terpene Content during the Growth of Cannabis sativa Plants from Different Chemotypes. J Nat Prod. 79: 324-331.

Amaducci, S., Colauzzi, M., Bellocchi, G., Cosentino, S.L., Pahkala, K., Stomph, T.J., et al. (2012) Evaluation of a phenological model for strategic decisions for hemp (Cannabis Sativa L.) biomass production across European sites.Ind Crops Prod. 37: 100-110.

Amaducci, S., Colauzzi, M., Bellocchi, G., and Venturi, G. (2008) Modelling post-emergent hemp phenology (Cannabis sativa L.): Theory and evaluation. Eur J Agron. 28: 90-102.

Amaducci, S., Scordia, D., Liu, F.H., Zhang, Q., Guo, H., Testa, G., et al. (2015) Key cultivation techniques for hemp in Europe and China. Ind Crops Prod. 68: 2-16.

Andre, C.M., Hausman, J.-F., and Guerriero, G. (2016) Cannabis sativa: The Plant of the Thousand and One Molecules. Front Plant Sci. 7.

Aranzana, M.J., Decroocq, V., Dirlewanger, E., Eduardo, I., Gao, Z.S., Gasic, K., et al. (2019) Prunus genetics and applications after de novo genome sequencing: achievements and prospects. Hortic Res. 6: 58.

Aryal, N., Orellana, D.F., and Bouie, J. (2019) Distribution of cannabinoid synthase genes in non-Cannabis organisms. J Cannabis Res. 1: 8.

Atalay, S., Jarocka-Karpowicz, I., and Skrzydlewska, E. (2019) Antioxidative and Anti-Inflammatory Properties of Cannabidiol. Antioxidants. 9.

Bachtrog, D., Mank, J.E., Peichel, C.L., Kirkpatrick, M., Otto, S.P., Ashman, T.-L., et al. (2014) Sex Determination: Why So Many Ways of Doing It? PLoS Biol. 12: e1001899.

van Bakel, H., Stout, J.M., Cote, A.G., Tallon, C.M., Sharpe, A.G., Hughes, T.R., et al. (2011) The draft genome and transcriptome of Cannabis sativa. Genome Biol. 12: R102.

Baram, L., Peled, E., Berman, P., Yellin, B., Besser, E., Benami, M., et al. (2019) The heterogeneity and complexity of Cannabis extracts as antitumor agents. Oncotarget. 10: 4091-4106.

Becker, A. (2020) A molecular update on the origin of the carpel. Curr Opin Plant Biol. 53: 15-22.

Behr, M., Legay, S., Žižková, E., Motyka, V., Dobrev, P.I., Hausman, J.F., et al. (2016) Studying secondary growth and bast fiber development: The hemp hypocotyl peeks behind the wall. Front Plant Sci. 7: 1-19.

Berman, P., Futoran, K., Lewitus, G.M., Mukha, D., Benami, M., Shlomi, T., et al. (2018) A new ESI-LC/MS approach for comprehensive metabolic profiling of phytocannabinoids in Cannabis. Sci Rep. 8: 14280.

Bhattarai, J.H., Surya P., and Midmore, D.J. (2014) Effect of industrial hemp (Cannabis sativa L) planting density on weed suppression, crop growth, physiological responses, and fibre yield in the subtropics. Renew Bioresour. 2: 1-1.

Blasco-Benito, S., Seijo-Vila, M., Caro-Villalobos, M., Tundidor, I., Andradas, C., García-Taboada, E., et al. (2018) Appraising the "entourage effect": Antitumor action of a pure cannabinoid versus a botanical drug preparation in preclinical models of breast cancer. Biochem Pharmacol. 157: 285-293.

Blümel, M., Dally, N., and Jung, C. (2015) Flowering time regulation in crops-what did we learn from Arabidopsis? Curr Opin Biotechnol. 32: 121-129.

Bócsa, I., and Karus, M. (1998) The cultivation of hemp: botany, varieties, cultivation and harvesting, Hemptech, Sebastopol, CA95473, California. 
Booth, J.K., and Bohlmann, J. (2019) Terpenes in Cannabis sativa - From plant genome to humans. Plant Sci. 284: $67-72$.

Booth, J.K., Yuen, M.M.S., Jancsik, S., Madilao, L., Page, J., and Bohlmann, J. (2020) Terpene Synthases and Terpene Variation in Cannabis sativa. Plant Physiol.

Borthwick, H.A., and Scully, N.J. (1954) Photoperiodic Responses of Hemp. Bot Gaz. 116: 14-29.

Boualem, A., Troadec, C., Camps, C., Lemhemdi, A., Morin, H., Sari, M.-A., et al. (2015) A cucurbit androecy gene reveals how unisexual flowers develop and dioecy emerges. Science. 350: 688-691.

Braich, S., Baillie, R.C., Jewell, L.S., Spangenberg, G.C., and Cogan, N.O.I. (2019) Generation of a Comprehensive Transcriptome Atlas and Transcriptome Dynamics in Medicinal Cannabis. Sci Rep. 9: 16583.

Brunetti, P., Faro, A.F.L., Pirani, F., Berretta, P., Pacifici, R., Pichini, S., et al. (2020) Pharmacology and legal status of cannabidiol. Ann Ist Super Sanità. 56.

Buckler, E.S., Holland, J.B., Bradbury, P.J., Acharya, C.B., Brown, P.J., Browne, C., et al. (2009) The Genetic Architecture of Maize Flowering Time.Science. 325: 714-718.

Burton, J.N., Adey, A., Patwardhan, R.P., Qiu, R., Kitzman, J.O., and Shendure, J. (2013) Chromosomescale scaffolding of de novo genome assemblies based on chromatin interactions. Nat Biotechnol. 31: 11191125 .

Cao, D., Takeshima, R., Zhao, C., Liu, B., Jun, A., and Kong, F. (2017) Molecular mechanisms of flowering under long days and stem growth habit in soybean. $J$ Exp Bot. 68: 1873-1884.

Carus, M., Kauffmann, A., Hobson, J., and Bertucelli, S. (2013) The European Hemp Industry: Cultivation, processing and applications for fibres, shivs and seeds.European Industrial Hemp Association.

Cascini, F., Farcomeni, A., Migliorini, D., Baldassarri, L., Boschi, I., Martello, S., et al. (2019) Highly Predictive Genetic Markers Distinguish Drug-Type from Fiber-Type Cannabis sativa L. Plants. 8: 496.

Chailakhyan, M.Kh., and Timiriazev, K.A. (1979) GENETIC AND HORMONAL REGULATION OF GROWTH, FLOWERING, AND SEX EXPRESSION IN PLANTS. Am J Bot. 66: 717-736.

Charlesworth, D. (2016) Plant Sex Chromosomes. Annu Rev Plant Biol. 67: 397-420.

Chiang, L., and Abdullah, M.A. (2007) Enhanced anthraquinones production from adsorbent-treated Morinda elliptica cell suspension cultures in production medium strategy. Process Biochem. 42: 757-763.

Citterio, S., Santagostino, A., Fumagalli, P., Prato, N., Ranalli, P., and Sgorbati, S. (2003) Heavy metal tolerance and accumulation of Cd, Cr and Ni by Cannabis sativa L. Plant Soil. 256: 243-252.

Clarke, R.C., and Merlin, M.D. (2016) Cannabis Domestication, Breeding History, Present-day Genetic Diversity, and Future Prospects. Crit Rev Plant Sci. 35: 293-327.

Claros, M.G., Bautista, R., Guerrero-Fernández, D., Benzerki, H., Seoane, P., and Fernández-Pozo, N. (2012) Why Assembling Plant Genome Sequences Is So Challenging. Biology. 1: 439-459.

Cole, L.W., Guo, W., Mower, J.P., and Palmer, J.D. (2018) High and Variable Rates of Repeat-Mediated Mitochondrial Genome Rearrangement in a Genus of Plants. Mol Biol Evol. 35: 2773-2785.

Collet-Foucault, F., Miriel, J., Institut national des sciences appliquées de Rennes, and Génie Civil et Urbanisme (2004) Caractérisation hydrique et thermique de matériaux de génie civil à faibles impacts environnementaux. Institut National des Sciences Appliquées RENNES-INSA.

Copley, T.R., Duceppe, M.O., and O'Donoughue, L.S. (2018) Identification of novel loci associated with maturity and yield traits in early maturity soybean plant introduction lines. BMC Genomics. 19: 1-12. 
Corbesier, L., Vincent, C., Jang, S., Fornara, F., Fan, Q., Searle, I., et al. (2007) FT protein movement contributes to long-distance signaling in floral induction of Arabidopsis. Science. 316: 1030-1033.

Cosentino, S.L., Testa, G., Scordia, D., and Copani, V. (2012) Sowing time and prediction of flowering of different hemp (Cannabis sativa L.) genotypes in southern Europe. Ind Crops Prod. 37: 20-33.

Crippa, J.A.S., Derenusson, G.N., Ferrari, T.B., Wichert-Ana, L., Duran, F.L., Martin-Santos, R., et al. (2011) Neural basis of anxiolytic effects of cannabidiol (CBD) in generalized social anxiety disorder: a preliminary report. J Psychopharmacol (Oxf). 25: 121-130.

Das, L., Liu, E., Saeed, A., Williams, D.W., Hu, H., Li, C., et al. (2017) Industrial hemp as a potential bioenergy crop in comparison with kenaf, switchgrass and biomass sorghum. Bioresour Technol. 244: 641649.

Davila, J.I., Arrieta-Montiel, M.P., Wamboldt, Y., Cao, J., Hagmann, J., Shedge, V., et al. (2011) Doublestrand break repair processes drive evolution of the mitochondrial genome in Arabidopsis. BMC Biol. 9: 64 .

Dayanandan, P., and Kaufman, P.B. (1976) TRICHOMES OF CANNABIS SATIVA L. (CANNABACEAE).Am J Bot. 63: 578-591.

Deiana, S. (2017) Potential Medical Uses of Cannabigerol: A Brief Overview. InHandbook of Cannabis and Related Pathologies. pp. 958-967 Academic Press.

Devinsky, O., Cross, J.H., Laux, L., Marsh, E., Miller, I., Nabbout, R., et al. (2017) Trial of Cannabidiol for Drug-Resistant Seizures in the Dravet Syndrome.N Engl J Med. 376: 2011-2020.

Divashuk, M.G., Alexandrov, O.S., Razumova, O.V., Kirov, I.V., and Karlov, G.I. (2014) Molecular Cytogenetic Characterization of the Dioecious Cannabis sativa with an XY Chromosome Sex Determination System. PLOS ONE. 9: e85118.

Elhamamsy, A.R. (2016) DNA methylation dynamics in plants and mammals: overview of regulation and dysregulation. Cell Biochem Funct. 34: 289-298.

Endress, P.K. (1992) Evolution and Floral Diversity: The Phylogenetic Surroundings of Arabidopsis and Antirrhinum. Int J Plant Sci. 153: S106-S122.

Endress, P.K. (2011) Evolutionary diversification of the flowers in angiosperms. Am J Bot. 98: 370-396.

Farag, S., and Kayser, O. (2015) Cannabinoids Production by Hairy Root Cultures of Cannabis sativa L. Am J Plant Sci. 06: 1874-1884.

Faux, A.-M., Berhin, A., Dauguet, N., and Bertin, P. (2014) Sex chromosomes and quantitative sex expression in monoecious hemp (Cannabis sativa L.).Euphytica. 196: 183-197.

Faux, A.-M., Draye, X., Flamand, M.-C., Occre, A., and Bertin, P. (2016) Identification of QTLs for sex expression in dioecious and monoecious hemp (Cannabis sativa L.). Euphytica. 209: 357-376.

Fellermeier, M., and Zenk, M.H. (1998) Prenylation of olivetolate by a hemp transferase yields cannabigerolic acid, the precursor of tetrahydrocannabinol.FEBS Lett. 427: 283-285.

Finnan, J., and Burke, B. (2013) Nitrogen fertilization to optimize the greenhouse gas balance of hemp crops grown for biomass. GCB Bioenergy. 5: 701-712.

Finnan, J., and Styles, D. (2013) Hemp: A more sustainable annual energy crop for climate and energy policy. Energy Policy. 58: 152-162.

Flores-Sanchez, I.J., Peč, J., Fei, J., Choi, Y.H., Dušek, J., and Verpoorte, R. (2009) Elicitation studies in cell suspension cultures of Cannabis sativa L.J Biotechnol. 143: 157-168. 
Fornara, F., de Montaigu, A., and Coupland, G. (2010) SnapShot: Control of Flowering in Arabidopsis. Cell. 141: 550-550.e2.

Fraguas-Sánchez, A.I., and Torres-Suárez, A.I. (2018) Medical Use of Cannabinoids.Drugs. 78: 1665-1703.

Freeman, D.C., Harper, K.T., and Charnov, E.L. (1980) Sex change in plants: Old and new observations and new hypotheses. Oecologia. 47: 222-232.

Freeman, T.P., Hindocha, C., Green, S.F., and Bloomfield, M.A.P. (2019) Medicinal use of cannabis based products and cannabinoids. BMJ. 11141.

Furr, M., and Mahlberg, P.G. (1981) Histochemical Analyses of Laticifers and Glandular Trichomes in Cannabis sativa. J Nat Prod. 44: 153-159.

Gage, J.L., Monier, B., Giri, A., Buckler, E.S., and Buckler, E.S. (2020) Ten Years of the maize Nested Association Mapping Population : Impact, Limitations, and Future Directions. Plant Cell. 32: 2083-2093.

Gai, Q.-Y., Jiao, J., Luo, M., Wei, Z.-F., Zu, Y.-G., Ma, W., et al. (2015) Establishment of Hairy Root Cultures by Agrobacterium Rhizogenes Mediated Transformation of Isatis Tinctoria L. for the Efficient Production of Flavonoids and Evaluation of Antioxidant Activities. PLOS ONE. 10.

Gallily, R., Yekhtin, Z., and Hanuš, L.O. (2015) Overcoming the Bell-Shaped Dose-Response of Cannabidiol by Using Cannabis Extract Enriched in Cannabidiol.Pharmacol Pharm. 06: 75-85.

Gamble, T., and Zarkower, D. (2012) Sex determination. Curr Biol. 22: R257-R262.

Gao, C., Cheng, C., Zhao, L., Yu, Y., Tang, Q., Xin, P., et al. (2018) Genome-Wide Expression Profiles of Hemp (Cannabis sativa L.) in Response to Drought Stress. Int J Genomics. 2018: 1-13.

Gao, S., Wang, B., Xie, S., Xu, X., Zhang, J., Pei, L., et al. (2020) A high-quality reference genome of wild Cannabis sativa. Hortic Res. 7.

Gaudinier, A., and Blackman, B.K. (2020) Evolutionary processes from the perspective of flowering time diversity. New Phytol. 225: 1883-1898.

Grassa, C.J., Wenger, J.P., Dabney, C., Poplawski, S.G., Motley, S.T., Michael, T.P., et al. (2018) A complete Cannabis chromosome assembly and adaptive admixture for elevated cannabidiol (CBD) content. bioRxiv.

Grassi, G., and McPartland, J.M. (2017) Chemical and Morphological Phenotypes in Breeding of Cannabis sativa L. In Cannabis Sativa L. - Botany and Biotechnology. Edited by Chandra, S., Lata, H., and ElSohly, M.A. pp. 137-160 Springer International Publishing, Cham.

Guerriero, G., Behr, M., Legay, S., Mangeot-Peter, L., Zorzan, S., Ghoniem, M., et al. (2017) Transcriptomic profiling of hemp bast fibres at different developmental stages. Sci Rep. 7.

Hall, J., Bhattarai, S.P., and Midmore, D.J. (2012) Review of Flowering Control in Industrial Hemp. J Nat Fibers. 9: 23-36.

Hammond, C.T., and Mahlberg, P.G. (1973) MORPHOLOGY OF GLANDULAR HAIRS OF CANNABIS SATIVA FROM SCANNING ELECTRON MICROSCOPY. Am J Bot. 60: 524-528.

Hao, Z., Lv, D., Ge, Y., Shi, J., Weijers, D., Yu, G., et al. (2020) RIdeogram: drawing SVG graphics to visualize and map genome-wide data on the idiograms.PeerJ Comput Sci. 6: e251.

He, J., Zhao, X., Laroche, A., Lu, Z.-X., Liu, H., and Li, Z. (2014) Genotyping-by-sequencing (GBS), an ultimate marker-assisted selection (MAS) tool to accelerate plant breeding. Front Plant Sci. 5: 484.

Hemp insulation: rolls and panels [WWW Document] (2017) . Technichanvre. URL http://www.technichanvre.com/1411-2/1499-2/hemp-insulationrolls-and-panels/ (accessed 8.5.20). 
Hepworth, S.R., Valverde, F., Ravenscroft, D., Mouradov, A., and Coupland, G. (2002) Antagonistic regulation of flowering-time gene SOC1 by CONSTANS and FLC via separate promoter motifs. EMBO J. 21: 4327-4337.

Heslop-Harrison, J. (1956) Auxin and Sexuality in Cannabis sativa. Physiol Plant. 9: 588-597.

Heslop-Harrison, J. (1957) The experimental modification of sex expression in flowering plants. Biol Rev. 32: $38-90$.

Hill, C.B., and Li, C. (2016) Genetic architecture of flowering phenology in cereals and opportunities for crop improvement. Front Plant Sci. 7: 1906-1906.

Holland, T., Sack, M., Rademacher, T., Schmale, K., Altmann, F., Stadlmann, J., et al. (2010) Optimal nitrogen supply as a key to increased and sustained production of a monoclonal full-size antibody in BY-2 suspension culture. Biotechnol. Bioeng. 107: 278-289.

Izzo, A.A., Borrelli, F., Capasso, R., Di Marzo, V., and Mechoulam, R. (2009) Non-psychotropic plant cannabinoids: new therapeutic opportunities from an ancient herb. Trends Pharmacol Sci. 30: 515-527.

James, E. and Lee, J.M. (2006) Loss and recovery of protein productivity in genetically modified plant cell lines. Plant Cell Rep. 25: 723-727.

Janatová, A., Fraňková, A., Tlustoš, P., Hamouz, K., Božik, M., and Klouček, P. (2018) Yield and cannabinoids contents in different cannabis ( Cannabis sativa L.) genotypes for medical use. Ind Crops Prod. 112: 363-367.

Jenkins, C., and Orsburn, B. (2019) The First Publicly Available Annotated Genome for Cannabis plants. bioRxiv.

Jiang, B., Zhang, S., Song, W., Khan, M.A.A., Sun, S., Zhang, C., et al. (2019) Natural variations of FT family genes in soybean varieties covering a wide range of maturity groups. BMC Genomics. 20: 1-16.

Jiao, J., Gai, Q.-Y., Fu, Y.-J., Ma, W., Peng, X., Tan, S.-N., et al. (2014) Efficient production of isoflavonoids by Astragalus membranaceus hairy root cultures and evaluation of antioxidant activities of extracts. J Agric Food Chem. 62: 12649-12658.

Jiao, W.-B., and Schneeberger, K. (2017) The impact of third generation genomic technologies on plant genome assembly. Curr Opin Plant Biol. 36: 64-70.

Jin, J., Yang, M., Fritsch, P.W., Velzen, R., Li, D., and Yi, T. (2020) Born migrators: Historical biogeography of the cosmopolitan family Cannabaceae. J Syst Evol. 58: 461-473.

Johnson, J.R., Burnell-Nugent, M., Lossignol, D., Ganae-Motan, E.D., Potts, R., and Fallon, M.T. (2010) Multicenter, Double-Blind, Randomized, Placebo-Controlled, Parallel-Group Study of the Efficacy, Safety, and Tolerability of THC:CBD Extract and THC Extract in Patients with Intractable Cancer-Related Pain. J Pain Symptom Manage. 39: 167-179.

Jung, C., and Müller, A.E. (2009) Flowering time control and applications in plant breeding. Trends Plant Sci. 14: 563-573.

Kim, E.-S., and Mahlberg, P.G. (1991) SECRETORY CAVITY DEVELOPMENT IN GLANDULAR TRICHOMES OF CANNABIS SATIVA L. (CANNABACEAE). Am J Bot. 78: 220-229.

Kinnane, O. (2020) Calculating the Cost of Building - Obsolescence, Architecture and the Climate Crisis. Archit Irel.

Kinnane, O., Reilly, A., Grimes, J., Walker, R., and Pavia, S. (2016) Acoustic absorption of hemp-lime construction. Constr Build Mater . 122: 674-682. 
Kirchhoff, J., Raven, N., Boes, A., Roberts, J.L., Russell, S., Treffenfeldt, W., et al. (2012) Monoclonal tobacco cell lines with enhanced recombinant protein yields can be generated from heterogeneous cell suspension cultures by flow sorting. Plant Biotechnol. J.10: 936-944.

Klose, C., Nagy, F., and Schäfer, E. (2020) Thermal Reversion of Plant Phytochromes.Mol Plant . 13: 386-397.

Knoop, V. (2004) The mitochondrial DNA of land plants: peculiarities in phylogenetic perspective. Curr Genet. 46: 123-139.

Komiya, M., Takeuchi, T., and Harada, E. (2006) Lemon oil vapor causes an anti-stress effect via modulating the 5-HT and DA activities in mice. Behav Brain Res. 172: 240-249.

Kovalchuk, I., Pellino, M., Rigault, P., van Velzen, R., Ebersbach, J., Ashnest, J.R., et al. (2020) The Genomics of Cannabis and Its Close Relatives.Annu Rev Plant Biol. 71: 713-739.

Kraszkiewicz, A., Kachel, M., Parafiniuk, S., Zaj, G., Niedziółka, I., and Sprawka, M. (2019) Assessment of the Possibility of Using Hemp Biomass (Cannabis Sativa L.) for Energy Purposes: A Case Study. Appl Sci. 9 .

Krizek, B.A., and Fletcher, J.C. (2005) Molecular mechanisms of flower development: an armchair guide. Nat Rev Genet. 6: 688-698.

Langer, S.M., Longin, C.F.H., and Würschum, T. (2014) Flowering time control in European winter wheat. Front Plant Sci. 5: 1-11.

Laverty, K.U., Stout, J.M., Sullivan, M.J., Shah, H., Gill, N., Holbrook, L., et al. (2019) A physical and genetic map of Cannabis sativa identifies extensive rearrangements at the THC/CBD acid synthase loci. Genome Res. 29: 146-156.

Lee, J., Oh, M., Park, H., and Lee, I. (2008) SOC1 translocated to the nucleus by interaction with AGL24 directly regulates LEAFY. Plant $J .55:$ 832-843.

Legris, M., Ince, Y.Ç., and Fankhauser, C. (2019) Molecular mechanisms underlying phytochrome-controlled morphogenesis in plants. Nat Commun. 10: 1-15.

Leme, F.M., Schönenberger, J., Staedler, Y.M., and Teixeira, S.P. (2020) Comparative floral development reveals novel aspects of structure and diversity of flowers in Cannabaceae. Bot J Linn Soc. 193: 64-83.

Li, D., Sheng, Y., Niu, H., and Li, Z. (2019) Gene Interactions Regulating Sex Determination in Cucurbits. Front Plant Sci. 10: 1231.

Li, R., Hu, F., Li, B., Zhang, Y., Chen, M., Fan, T., et al. (2020) Whole genome bisulfite sequencing methylome analysis of mulberry ( Morus alba ) reveals epigenome modifications in response to drought stress. Sci Rep. 10: 1-17.

Lieberman-Aiden, E., van Berkum, N.L., Williams, L., Imakaev, M., Ragoczy, T., Telling, A., et al. (2009) Comprehensive mapping of long-range interactions reveals folding principles of the human genome. Science. 326: 289-293.

Lisson, S.N., Mendham, N.J., and Carberry, P.S. (2000) Development of a hemp (Cannabis sativa L.) simulation model 2.The flowering response of two hemp cultivars to photoperiod. Aust J Exp Agric. 40: 413.

Liu, J., Qiao, Q., Cheng, X., Du, G., Deng, G., Zhao, M., et al. (2016) Transcriptome differences between fiber-type and seed-type Cannabis sativa variety exposed to salinity. Physiol Mol Biol Plants. 22: 429-443.

Livingston, S.J., Quilichini, T.D., Booth, J.K., Wong, D.C.J., Rensing, K.H., Laflamme-Yonkman, J., et al. (2020) Cannabis glandular trichomes alter morphology and metabolite content during flower maturation. Plant J. 101: 37-56. 
Lu, S., Zhao, X., Hu, Y., Liu, S., Nan, H., Li, X., et al. (2017) Natural variation at the soybean J locus improves adaptation to the tropics and enhances yield.Nat Genet. 49: 773-779.

Luo, X., Reiter, M.A., d'Espaux, L., Wong, J., Denby, C.M., Lechner, A., et al. (2019) Complete biosynthesis of cannabinoids and their unnatural analogues in yeast.Nature. 567: 123-126.

Lynch, R.C., Vergara, D., Tittes, S., White, K., Schwartz, C.J., Gibbs, M.J., et al. (2016) Genomic and Chemical Diversity in Cannabis. Crit Rev Plant Sci. 35: 349-363.

Magallon, S., Gomez-Acevedo, S., Sanchez-Reyes, L.L., and Hernandez-Hernandez, T. (2015) A metacalibrated time-tree documents the early rise of flowering plant phylogenetic diversity. New Phytol. 207: $437-453$.

Malik, S., Hossein Mirjalili, M., Fett-Neto, A.G., Mazzafera, P., and Bonfill, M. (2013) Living between two worlds: two-phase culture systems for producing plant secondary metabolites. Crit Rev Biotechnol. 33: $1-22$.

McDaniel, B.K., and Binder, B.M. (2012) Ethylene Receptor 1 (ETR1) Is Sufficient and Has the Predominant Role in Mediating Inhibition of Ethylene Responses by Silver in Arabidopsis thaliana. J Biol Chem. 287: 26094-26103.

McKernan, K., Helbert, Y., Kane, L.T., Ebling, H., Zhang, L., Liu, B., et al. (2018) Cryptocurrencies and Zero Mode Wave guides: An unclouded path to a more contiguous Cannabis sativa L. genome assembly. OSFPREPRINTS.

McKernan, K.J., Helbert, Y., Kane, L.T., Ebling, H., Zhang, L., Liu, B., et al. (2020) Sequence and annotation of 42 cannabis genomes reveals extensive copy number variation in cannabinoid synthesis and pathogen resistance genes (preprint). Genomics.

Mead, A. (2017) The legal status of cannabis (marijuana) and cannabidiol (CBD) under U.S. law. Epilepsy Behav. 70: 288-291.

Meier, C., and Mediavilla, V. (1998) Factors influencing the yield and the quality of hemp essential oil. $J$ Int Hemp Assoc. 5: 16-20.

de Meijer, E.P.M., Bagatta, M., Carboni, A., Crucitti, P., Moliterni, V.M.C., Ranalli, P., et al. (2003) The inheritance of chemical phenotype in Cannabis sativa L. Genetics. 163: 335-346.

de Meijer, E.P.M., and Hammond, K.M. (2005) The inheritance of chemical phenotype in Cannabis sativa L. (II): Cannabigerol predominant plants. Euphytica. 145: 189-198.

de Meijer, E.P.M., Hammond, K.M., and Micheler, M. (2009a) The inheritance of chemical phenotype in Cannabis sativa L. (III): variation in cannabichromene proportion. Euphytica. 165: 293-311.

de Meijer, E.P.M., Hammond, K.M., and Sutton, A. (2009b) The inheritance of chemical phenotype in Cannabis sativa L. (IV): cannabinoid-free plants.Euphytica. 168: 95-112.

Michael, T.P., and VanBuren, R. (2020) Building near-complete plant genomes. Curr Opin Plant Biol., Genome studies and molecular genetics 54: 26-33.

Mohan Ram, H.Y., and Sett, R. (1982) Induction of fertile male flowers in genetically female Cannabis sativa plants by silver nitrate and silver thiosulphate anionic complex. Theor Appl Genet. 62: 369-375.

Moliterni, V.M.C., Cattivelli, L., Ranalli, P., and Mandolino, G. (2004) The sexual differentiation of Cannabis sativa L.: A morphological and molecular study. Euphytica. 140: 95-106.

Montenegro, J.D., Golicz, A.A., Bayer, P.E., Hurgobin, B., Lee, H., Chan, C.-K.K., et al. (2017) The pangenome of hexaploid bread wheat. Plant J. 90: 1007-1013. 
Moon, K.-B., Park, J.-S., Park, Y.-I., Song, I.-J., Lee, H.-J., Cho, H.S., et al. (2020) Development of Systems for the Production of Plant-Derived Biopharmaceuticals. Plants. 9.

Moreno-Sanz, G. (2016) Can You Pass the Acid Test? Critical Review and Novel Therapeutic Perspectives of $\Delta 9$-Tetrahydrocannabinolic Acid A.Cannabis Cannabinoid Res. 1: 124-130.

Morimoto, S., Komatsu, K., Taura, F., and Shoyama, Y. (1997) Enzymological Evidence for Cannabichromenic Acid Biosynthesis. J Nat Prod. 60: 854-857.

Mountford, P.N.G. (2010) The Taxol@ Story - Development of a Green Synthesis via Plant Cell Fermentation. In Green Chemistry in the Pharmaceutical Industry. pp. 145-160.

Murari, G., Puccini, A.M., Sanctis, R. de (Bologna U. (Italy) I. di F., and Lombardi, S. (Istituto di I. e P. (1983) Influence of environmental conditions on tetrahydrocannabinol (delta -9-TCH) in different cultivars on Cannabis sativa L. Fitoterapia. 54: 195-201.

Mustafa, N.R., de Winter, W., van Iren, F., and Verpoorte, R. (2011) Initiation, growth and cryopreservation of plant cell suspension cultures. Nat Protoc. 6: 715-742.

Namdar, D., Voet, H., Ajjampura, V., Nadarajan, S., Mayzlish-Gati, E., Mazuz, M., et al. (2019) Terpenoids and Phytocannabinoids Co-Produced in Cannabis Sativa Strains Show Specific Interaction for Cell Cytotoxic Activity.Molecules. 24: 3031.

Nan, H., Cao, D., Zhang, D., Li, Y., Lu, S., Tang, L., et al. (2014) GmFT2a and GmFT5a redundantly and differentially regulate flowering through interaction with and upregulation of the bZIP transcription factor GmFDL19 in soybean. PLoS ONE. 9.

Nelson, C.H. (1944) Growth Responses of Hemp To Differential Soil and Air Temperatures.Plant Physiol. 19: 294-309.

Niederhuth, C.E., Bewick, A.J., Ji, L., Alabady, M.S., Kim, K.D., Li, Q., et al. (2016) Widespread natural variation of DNA methylation within angiosperms. Genome Biol. 17: 194.

Ogawa, Y., Sakurai, N., Oikawa, A., Kai, K., Morishita, Y., Mori, K., et al. (2012) High-throughput cryopreservation of plant cell cultures for functional genomics. Plant Cell Physiol. 53: 943-952.

Oh, H., Seo, B., Lee, S., Ahn, D.-H., Jo, E., Park, J.-K., et al. (2016) Two complete chloroplast genome sequences of Cannabis sativa varieties. Mitochondrial DNA Part A . 27: 2835-2837.

Ono, N.N., and Tian, L. (2011) The multiplicity of hairy root cultures: Prolific possibilities. Plant Sci. 180: 439-446.

Onofri, C., de Meijer, E.P.M., and Mandolino, G. (2015) Sequence heterogeneity of cannabidiolic- and tetrahydrocannabinolic acid-synthase in Cannabis sativa L. and its relationship with chemical phenotype.Phytochemistry. 116: 57-68.

Pacifico, D., Miselli, F., Carboni, A., Moschella, A., and Mandolino, G. (2008) Time course of cannabinoid accumulation and chemotype development during the growth of Cannabis sativa L. Euphytica. 160: 231-240.

Pacifico, D., Miselli, F., Micheler, M., Carboni, A., Ranalli, P., and Mandolino, G. (2006) Genetics and Marker-assisted Selection of the Chemotype in Cannabis sativa L. Mol Breed. 17: 257-268.

Page, J.E., and Nagel, J. (2006) Biosynthesis of Terpenophenolic Metabolites in Hop and Cannabis. In Recent Advances in Phytochemistry. pp. 179-210 Elsevier.

Palmer, J.D., and Herbon, L.A. (1988) Plant mitochondrial DNA evolved rapidly in structure, but slowly in sequence. J Mol Evol. 28: 87-97.

Pamplona, F.A., da Silva, L.R., and Coan, A.C. (2018) Potential Clinical Benefits of CBD-Rich Cannabis Extracts Over Purified CBD in Treatment-Resistant Epilepsy: Observational Data Meta-analysis. Front 
Neurol. 9: 759 .

Parker, J.S., and Clark, M.S. (1991) Dosage sex-chromosome systems in plants. Plant Sci. 80: 79-92.

Patra, N., and Srivastava, A.K. (2014) Enhanced production of artemisinin by hairy root cultivation of Artemisia annua in a modified stirred tank reactor.Appl Biochem Biotechnol. 174: 2209-2222.

Paul, M.J., Thangaraj, H., and Ma, J.K.-C. (2015) Commercialization of new biotechnology: a systematic review of 16 commercial case studies in a novel manufacturing sector. Plant Biotechnol J. 13: 1209-1220.

Pavlovic, R., Panseri, S., Giupponi, L., Leoni, V., Citti, C., Cattaneo, C., et al. (2019) Phytochemical and Ecological Analysis of Two Varieties of Hemp (Cannabis sativa L.) Grown in a Mountain Environment of Italian Alps. Front Plant Sci. 10: 1265.

Pawełkowicz, M.E., Skarzyńska, A., Plader, W., and Przybecki, Z. (2019) Genetic and molecular bases of cucumber (Cucumis sativus L.) sex determination. Mol Breed. 39: 50.

Pertwee, R.G. (2014) Handbook of Cannabis. Oxford University Press, Oxford, UK.

Piccinelli, A.C., Santos, J.A., Konkiewitz, E.C., Oesterreich, S.A., Formagio, A.S.N., Croda, J., et al. (2015) Antihyperalgesic and antidepressive actions of (R)-(+)-limonene, $\alpha$-phellandrene, and essential oil from Schinus terebinthifolius fruits in a neuropathic pain model. Nutr Neurosci. 18: 217-224.

Pisupati, R., Vergara, D., and Kane, N.C. (2018) Diversity and evolution of the repetitive genomic content in Cannabis sativa. BMC Genomics. 19: 156.

Poleszak, E., Wośko, S., Sławińska, K., Szopa, A., Wróbel, A., and Serefko, A. (2018) Cannabinoids in depressive disorders. Life Sci. 213: 18-24.

Pollastro, F., Caprioglio, D., Del Prete, D., Rogati, F., Minassi, A., Taglialatela-Scafati, O., et al. (2018a) Cannabichromene. Nat Prod Commun. 13: 1934578X1801300.

Pollastro, F., Minassi, A., and Fresu, L.G. (2018b) Cannabis Phenolics and their Bioactivities. Curr Med Chem. 25: 1160-1185.

Potter, D.J. (2014) A review of the cultivation and processing of cannabis (Cannabis sativa L.) for production of prescription medicines in the UK. Drug Test Anal. 6: 31-38.

Prade, T., Svensson, S.E., Andersson, A., and Mattsson, J.E. (2011) Biomass and energy yield of industrial hemp grown for biogas and solid fuel. Biomass Bioenergy. 35: 3040-3049.

Prade, T., Svensson, S.E., and Mattsson, J.E. (2012) Energy balances for biogas and solid biofuel production from industrial hemp. Biomass Bioenergy. 40: 36-52.

Prentout, D., Razumova, O., Rhoné, B., Badouin, H., Henri, H., Feng, C., et al. (2020) An efficient RNAseq-based segregation analysis identifies the sex chromosomes of Cannabis sativa. Genome Res. 30: 164-172.

Putterill, J., Robson, F., Lee, K., Simon, R., and Coupland, G. (1995) The CONSTANS gene of arabidopsis promotes flowering and encodes a protein showing similarities to zinc finger transcription factors. Cell. 80: $847-857$.

Ram, H.Y.M., and Jaiswal, V.S. (1970) Induction of female flowers on male plants ofCannabis Sativa L. by 2-chloroethanephos-phonic acid.Experientia. 26: 214-216.

Ram, H.Y.M., and Jaiswal, V.S. (1972) Induction of male flowers on female plants of Cannabis sativa by gibberellins and its inhibition by abscisic acid.Planta. 105: 263-266.

Raman, V., Lata, H., Chandra, S., Khan, I.A., and ElSohly, M.A. (2017) Morpho-Anatomy of Marijuana (Cannabis sativa L.). In Cannabis Sativa L. - Botany and Biotechnology. Edited by Chandra, S., Lata, H., and ElSohly, M.A. pp. 123-136 Springer International Publishing, Cham. 
Razumova, O.V., Alexandrov, O.S., Divashuk, M.G., Sukhorada, T.I., and Karlov, G.I. (2016) Molecular cytogenetic analysis of monoecious hemp (Cannabis sativa L.) cultivars reveals its karyotype variations and sex chromosomes constitution. Protoplasma. 253: 895-901.

Reed, J. (1914) Morphology of cannabis sativa L (Master of Science). University of Iowa.

Reilly, A., and Kinnane, O. (2017) Presented at the International Conference for Sustainable Design of the Built Environment London.

Reilly, A., Kinnane, O., Lesage, F.J., McGranaghan, G., Pavía, S., Walker, R., et al. (2019) The thermal diffusivity of hemplime, and a method of direct measurement. Constr Build Mater. 212: 707-715.

Renner, S.S. (2014) The relative and absolute frequencies of angiosperm sexual systems: Dioecy, monoecy, gynodioecy, and an updated online database. Am J Bot. 101: 1588-1596.

Rodriguez-Granados, N.Y., Ramirez-Prado, J.S., Veluchamy, A., Latrasse, D., Raynaud, C., Crespi, M., et al. (2016) Put your 3D glasses on: plant chromatin is on show. J Exp Bot. 67: 3205-3221.

Rogelj, J., Shindell, D., Jiang, K., Fifita, S., P. Forster, Ginzburg, V., et al. (2018) Chapter 2: Mitigation pathways compatible with $1.5^{\circ} \mathrm{C}$ in the context of sustainable development. In Global Warming of $1.5{ }^{\circ} \mathrm{C}$ an IPCC Special Report on the Impacts of Global Warming of $1.5{ }^{\circ} \mathrm{C}$ above Pre-Industrial Levels and Related Global Greenhouse Gas Emission Pathways, in the Context of Strengthening the Global Response to the Threat of Climate Change. Intergovernmental Panel on Climate Change.

Rudrappa, T., Neelwarne, B., and Aswathanarayana, R.G. (2004) In situ and Ex situ adsorption and recovery of betalains from hairy root cultures of Beta vulgaris. Biotechnol Prog. 20: 777-785.

Russo, E.B. (2011) Taming THC: potential cannabis synergy and phytocannabinoid-terpenoid entourage effects. Br J Pharmacol. 163: 1344-1364.

Russo, E.B. (2019) The Case for the Entourage Effect and Conventional Breeding of Clinical Cannabis: No "Strain," No Gain. Front Plant Sci. 9.

Sáez-Pérez, M.P., Brümmer, M., and Durán-Suárez, J.A. (2020) A review of the factors affecting the properties and performance of hemp aggregate concretes.J Build Eng. 31: 101323.

Sakamoto, K., Akiyama, Y., Fukui, K., Kamada, H., and Satoh, S. (1998) Characterization; Genome Sizes and Morphology of Sex Chromosomes in Hemp (Cannabis sativa L.).Cytologia (Tokyo). 63: 459-464.

Sakamoto, K., Ohmido, N., Fukui, K., Kamada, H., and Satoh, S. (2000) Site-specific accumulation of a LINE-like retrotransposon in a sex chromosome of the dioecious plant Cannabis sativa. Plant Mol Biol. 44: $723-732$.

Salentijn, E.M.J., Petit, J., and Trindade, L.M. (2019) The complex interactions between flowering behavior and fiber quality in hemp. Front Plant Sci. 10.

Salentijn, E.M.J., Zhang, Q., Amaducci, S., Yang, M., and Trindade, L.M. (2015) New developments in fiber hemp (Cannabis sativa L.) breeding. Ind Crops Prod. 68: 32-41.

Samach, A., Onouchi, H., Gold, S.E., Ditta, G.S., Schwarz-Sommer, Z., Yanofsky, M.F., et al. (2000) Distinct roles of constans target genes in reproductive development of Arabidopsis. Science. 288: 1613-1616.

Samanfar, B., Molnar, S.J., Charette, M., Schoenrock, A., Dehne, F., Golshani, A., et al. (2017) Mapping and identification of a potential candidate gene for a novel maturity locus, E10, in soybean. Theor Appl Genet. 130: 377-390.

Santos, A.P., Gaudin, V., Mozgová, I., Pontvianne, F., Schubert, D., Tek, A.L., et al. (2020) Tidying-up the plant nuclear space: domains, function, and dynamics.J Exp Bot. eraa282. 
Santos, R.B., Abranches, R., Fischer, R., Sack, M., and Holland, T. (2016) Putting the Spotlight Back on Plant Suspension Cultures. Front Plant Sci. 7.

Sawa, M., Nusinow, D.A., Kay, S.A., and Imaizumi, T. (2007) FKF1 and GIGANTEA Complex Formation Is Required for Day-Length Measurement in Arabidopsis.Science. 318: 261-266.

Sawler, J., Stout, J.M., Gardner, K.M., Hudson, D., Vidmar, J., Butler, L., et al. (2015) The Genetic Structure of Marijuana and Hemp. PLOS ONE. 10: e0133292.

Schaffner, J.H. (1923) The Influence of Relative Length of Daylight on the Reversal of Sex in Hemp. Ecology. 4: $323-334$.

Schilling, S., McCabe, P.F., and Melzer, R. (2020a) Love is in the air: ethylene and sex determination in Cucurbita pepo. J Exp Bot. 71: 4-6.

Schilling, S., Melzer, R., and McCabe, P.F. (2020b) Cannabis sativa. Curr Biol. 30: R8-R9.

Schilling, S., Pan, S., Kennedy, A., and Melzer, R. (2018) MADS-box genes and crop domestication: The jack of all traits. J Exp Bot. 69: 1447-1469.

Schmutz, J., Cannon, S.B., Schlueter, J., Ma, J., Mitros, T., Nelson, W., et al. (2010) Genome sequence of the palaeopolyploid soybean. Nature. 463: 178-183.

Searle, I., He, Y., Turck, F., Vincent, C., Fornara, F., Kröber, S., et al. (2006) The transcription factor FLC confers a flowering response to vernalization by repressing meristem competence and systemic signaling in Arabidopsis. Genes Dev. 20: 898-912.

Shea, A., Lawrence, M., and Walker, P. (2012) Hygrothermal performance of an experimental hemp-lime building. Constr Build Mater. 36: 270-275.

Shim, J.S., Kubota, A., and Imaizumi, T. (2017) Circadian clock and photoperiodic flowering in Arabidopsis: CONSTANS is a Hub for signal integration. Plant Physiol. 173: 5-15.

Shoyama, Yoshinari, Tamada, T., Kurihara, K., Takeuchi, A., Taura, F., Arai, S., et al. (2012) Structure and Function of [?]1-Tetrahydrocannabinolic Acid (THCA) Synthase, the Enzyme Controlling the Psychoactivity of Cannabis sativa.J Mol Biol. 423: 96-105.

Sirikantaramas, S., Morimoto, S., Shoyama, Yoshinari, Ishikawa, Y., Wada, Y., Shoyama, Yukihiro, et al. (2004) The gene controlling marijuana psychoactivity: molecular cloning and heterologous expression of Delta1-tetrahydrocannabinolic acid synthase from Cannabis sativa L.J Biol Chem. 279: 39767-39774.

Sirikantaramas, S., Taura, F., Tanaka, Y., Ishikawa, Y., Morimoto, S., and Shoyama, Y. (2005) Tetrahydrocannabinolic acid synthase, the enzyme controlling marijuana psychoactivity, is secreted into the storage cavity of the glandular trichomes. Plant Cell Physiol. 46: 1578-1582.

Śledziński, P., Zeyland, J., Słomski, R., and Nowak, A. (2018) The current state and future perspectives of cannabinoids in cancer biology. Cancer Med. 7: 765-775.

Small, E. (2015) Evolution and Classification of Cannabis sativa (Marijuana, Hemp) in Relation to Human Utilization. Bot Rev. 81: 189-294.

Small, E. (2018) Dwarf germplasm: the key to giant Cannabis hemp seed and cannabinoid crops. Genet Resour Crop Evol. 65: 1071-1107.

Small, E., and Beckstead, H.D. (1973) Cannabinoid Phenotypes in Cannabis sativa.Nature. 245: 147-148.

Soorni, A., Fatahi, R., Haak, D.C., Salami, S.A., and Bombarely, A. (2017) Assessment of Genetic Diversity and Population Structure in Iranian Cannabis Germplasm. Sci Rep. 7: 1-10.

Spitzer-Rimon, B., Duchin, S., Bernstein, N., and Kamenetsky, R. (2019) Architecture and Florogenesis in Female Cannabis sativa Plants. Front Plant Sci. 10: 350. 
Sykłowska-Baranek, K., Rymaszewski, W., Gaweł, M., Rokicki, P., Pilarek, M., Grech-Baran, M., et al. (2019) Comparison of elicitor-based effects on metabolic responses of Taxus $\times$ media hairy roots in perfluorodecalin-supported two-phase culture system. Plant Cell Rep. 38: 85-99.

Sytsma, K.J., Morawetz, J., Pires, J.C., Nepokroeff, M., Conti, E., Zjhra, M., et al. (2002) Urticalean rosids: circumscription, rosid ancestry, and phylogenetics based on rbcL, trnL-F, and ndhF sequences. Am J Bot. 89: 1531-1546.

Takeno, K. (2016) Stress-induced flowering: The third category of flowering response.J Exp Bot. 67: 49254934 .

Tang, K., Struik, P.C., Amaducci, S., Stomph, T.J., and Yin, X. (2017) Hemp (Cannabis sativa L.) leaf photosynthesis in relation to nitrogen content and temperature: implications for hemp as a bio-economically sustainable crop. GCB Bioenergy. 9: 1573-1587.

Tao, Y., Zhao, X., Mace, E., Henry, R., and Jordan, D. (2019) Exploring and Exploiting Pan-genomics for Crop Improvement. Mol Plant. 12: 156-169.

Taura, F., Morimoto, S., and Shoyama, Y. (1996) Purification and Characterization of Cannabidiolic-acid Synthase from Cannabis sativa L.: BIOCHEMICAL ANALYSIS OF A NOVEL ENZYME THAT CATALYZES THE OXIDOCYCLIZATION OF CANNABIGEROLIC ACID TO CANNABIDIOLIC ACID. $J$ Biol Chem. 271: 17411-17416.

Tekoah, Y., Shulman, A., Kizhner, T., Ruderfer, I., Fux, L., Nataf, Y., et al. (2015) Large-scale production of pharmaceutical proteins in plant cell culture-the protalix experience. Plant Biotechnol. J. 13: 1199-1208.

The Angiosperm Phylogeny Group (2016) An update of the Angiosperm Phylogeny Group classification for the orders and families of flowering plants: APG IV.Bot J Linn Soc. 181: 1-20.

Theissen, G., and Melzer, R. (2007) Molecular Mechanisms Underlying Origin and Diversification of the Angiosperm Flower. Ann Bot. 100: 603-619.

Thiele, E.A., Marsh, E.D., French, J.A., Mazurkiewicz-Beldzinska, M., Benbadis, S.R., Joshi, C., et al. (2018) Cannabidiol in patients with seizures associated with Lennox-Gastaut syndrome (GWPCARE4): a randomised, double-blind, placebo-controlled phase 3 trial. Lancet Lond Engl. 391: 1085-1096.

Toonen, M., Ribot, S., and Thissen, J. (2006) Yield of illicit indoor cannabis cultivation in the Netherlands. J Forensic Sci. 51: 1050-1054.

Toth, J.A., Stack, G.M., Cala, A.R., Carlson, C.H., Wilk, R.L., Crawford, J.L., et al. (2020) Development and validation of genetic markers for sex and cannabinoid chemotype in Cannabis sativa L. GCB Bioenergy. 12: $213-222$.

Turck, F., Fornara, F., and Coupland, G. (2008) Regulation and Identity of Florigen: FLOWERING LOCUS T Moves Center Stage. Annu Rev Plant Biol. 59: 573-594.

Turner, J.C., Hemphill, J.K., and Mahlberg, P.G. (1978) Quantitative Determination of Cannabinoids in Individual Glandular Trichomes of Cannabis Sativa L. (Cannabaceae). Am J Bot.

Ullisch, D.A., Müller, C.A., Maibaum, S., Kirchhoff, J., Schiermeyer, A., Schillberg, S., et al. (2012) Comprehensive characterization of two different Nicotiana tabacum cell lines leads to doubled GFP and HA protein production by media optimization. J. Biosci. Bioeng.113: 242-248.

Vanhove, W., Van Damme, P., and Meert, N. (2011) Factors determining yield and quality of illicit indoor cannabis (Cannabis spp.) production. Forensic Sci Int. 212: 158-163.

Varotto, S., Tani, E., Abraham, E., Krugman, T., Kapazoglou, A., Melzer, R., et al. (2020) Epigenetics: possible applications in climate-smart crop breeding.J Exp Bot. eraa188. 
Vasilev, N., Grömping, U., Lipperts, A., Raven, N., Fischer, R., and Schillberg, S. (2013) Optimization of BY-2 cell suspension culture medium for the production of a human antibody using a combination of fractional factorial designs and the response surface method.Plant Biotechnol. J. 11: 867-874.

Vergara, D., Baker, H., Clancy, K., Keepers, K.G., Mendieta, J.P., Pauli, C.S., et al. (2016) Genetic and Genomic Tools for Cannabis sativa. Crit Rev Plant Sci. 35: 364-377.

Vergara, D., Huscher, E.L., Keepers, K.G., Givens, R.M., Cizek, C.G., Torres, A., et al. (2019) Gene copy number is associated with phytochemistry in Cannabis sativa. AoB PLANTS. 11: plz074.

Vergara, D., White, K.H., Keepers, K.G., and Kane, N.C. (2015) The complete chloroplast genomes of Cannabis sativa and Humulus lupulus. Mitochondrial DNA Part A. 27: 3793-3794.

Wagner, H., and Ulrich-Merzenich, G. (2009) Synergy research: Approaching a new generation of phytopharmaceuticals. Phytomedicine. 16: 97-110.

Wahby, I., Caba, J.M., and Ligero, F. (2013) Agrobacterium infection of hemp (Cannabis sativa L.): establishment of hairy root cultures. J Plant Interact. 8: 312-320.

Walker, R., and Pavía, S. (2014) Moisture transfer and thermal properties of hemp-lime concretes. Constr Build Mater. 64: 270-276.

Warmke, H.E., and Davidson, H. (1944) Polyploidy investigations. In Carnegie Institution of Washington, Year Book No. 43. pp. 135-139.

Watanabe, S., Xia, Z., Hideshima, R., Tsubokura, Y., Sato, S., Yamanaka, N., et al. (2011) A map-based cloning strategy employing a residual heterozygous line reveals that the GIGANTEA gene is involved in soybean maturity and flowering. Genetics. 188: 395-407.

Wawrosch, C., Schwaiger, S., Stuppner, H., and Kopp, B. (2014) Lignan formation in hairy root cultures of Edelweiss (Leontopodium nivale ssp. alpinum (Cass.) Greuter). Fitoterapia. 97: 219-223.

Weathers, P.J., Towler, M.J., and Xu, J. (2010) Bench to batch: advances in plant cell culture for producing useful products. Appl Microbiol Biotechnol. 85: 1339-1351.

Weiblen, G.D., Wenger, J.P., Craft, K.J., ElSohly, M.A., Mehmedic, Z., Treiber, E.L., et al. (2015) Gene duplication and divergence affecting drug content in Cannabis sativa. New Phytol. 208: 1241-1250.

Welling, M.T., Shapter, T., Rose, T.J., Liu, L., Stanger, R., and King, G.J. (2016) A Belated Green Revolution for Cannabis: Virtual Genetic Resources to Fast-Track Cultivar Development. Front Plant Sci. 7 .

Westergaard, M. (1958) The Mechanism of Sex Determination in Dioecious Flowering Plants. InAdvances in Genetics. pp. 217-281 Elsevier.

Whiting, P.F., Wolff, R.F., Deshpande, S., Di Nisio, M., Duffy, S., Hernandez, A.V., et al. (2015) Cannabinoids for Medical Use: A Systematic Review and Meta-analysis. JAMA. 313: 2456-2473.

Wolfe, K.H., Li, W.H., and Sharp, P.M. (1987) Rates of nucleotide substitution vary greatly among plant mitochondrial, chloroplast, and nuclear DNAs. Proc Natl Acad Sci U S A. 84: 9054-9058.

Wu, J., and Lin, L. (2003) Enhancement of taxol production and release in Taxus chinensis cell cultures by ultrasound, methyl jasmonate and in situ solvent extraction. Appl Microbiol Biotechnol. 62: 151-155.

Xia, Z., Watanabe, S., Yamada, T., Tsubokura, Y., Nakashima, H., Zhai, H., et al. (2012) Positional cloning and characterization reveal the molecular basis for soybean maturity locus E1 that regulates photoperiodic flowering.Proc Natl Acad Sci U S A. 109.

Xie, T., Zheng, J.-F., Liu, S., Peng, C., Zhou, Y.-M., Yang, Q.-Y., et al. (2015) De Novo Plant Genome Assembly Based on Chromatin Interactions: A Case Study of Arabidopsis thaliana. Mol Plant. 8: 489-492. 
Xu, M., Xu, Z., Liu, B., Kong, F., Tsubokura, Y., Watanabe, S., et al. (2013) Genetic variation in four maturity genes affects photoperiod insensitivity and PHYA-regulated post-flowering responses of soybean. BMC Plant Biol. 13: 1-14.

Yang, M.-Q., van Velzen, R., Bakker, F.T., Sattarian, A., Li, D.-Z., and Yi, T.-S. (2013) Molecular phylogenetics and character evolution of Cannabaceae.Taxon. 62: 473-485.

Yoo, S.K., Chung, K.S., Kim, J., Lee, J.H., Hong, S.M., Yoo, S.J., et al. (2016) CONSTANS Activates SUPPRESSOR OF OVEREXPRESSION OF CONSTANS 1 through FLOWERING LOCUS T to Promote Flowering in Arabidopsis. Plant Physiol. 139: 770-778.

Zager, J.J., Lange, I., Srividya, N., Smith, A., and Lange, B.M. (2019) Gene Networks Underlying Cannabinoid and Terpenoid Accumulation in Cannabis.Plant Physiol. 180: 1877-1897.

Zanelati, T.V., Biojone, C., Moreira, F.A., Guimarães, F.S., and Joca, S.R.L. (2010) Antidepressant-like effects of cannabidiol in mice: possible involvement of 5-HT1A receptors. Br J Pharmacol. 159: 122-128.

Zhai, H., Lü, S., Liang, S., Wu, H., Zhang, X., Liu, B., et al. (2014) GmFT4, a homolog of FLOWERING LOCUS T, is positively regulated by E1 and functions as a flowering repressor in soybean. PLOS ONE. 9.

Zhang, H., Jin, J., Moore, M.J., Yi, T., and Li, D. (2018) Plastome characteristics of Cannabaceae. Plant Divers. 40: 127-137.

Zhang, L., Hu, J., Han, X., Li, J., Gao, Y., Richards, C.M., et al. (2019) A high-quality apple genome assembly reveals the association of a retrotransposon and red fruit colour. Nat Commun. 10: 1494.

Zhang, Q., Chen, X., Guo, H., Trindade, L.M., Salentijn, E.M.J., Guo, R., et al. (2018) Latitudinal Adaptation and Genetic Insights Into the Origins of Cannabis sativa L. Front Plant Sci. 9: 1876.

Zhang, S., Soltis, D.E., Yang, Y., Li, D., and Yi, T. (2011) Multi-gene analysis provides a well-supported phylogeny of Rosales. Mol Phylogenet Evol. 60: 21-28.

Zheng, Y., Wu, S., Bai, Y., Sun, H., Jiao, C., Guo, S., et al. (2019) Cucurbit Genomics Database (CuGenDB): a central portal for comparative and functional genomics of cucurbit crops. Nucleic Acids Res. 47: D1128D1136.

Zirpel, B., Kayser, O., and Stehle, F. (2018) Elucidation of structure-function relationship of THCA and CBDA synthase from Cannabis sativa L. J Biotechnol. 284: 17-26.

\section{Tables}

Table 1. Summary of the currently available Cannabis genome assemblies.

\section{Hosted file}

Schilling2020_Table.xlsx available at https://authorea.com/users/360584/articles/483690-thecream-of-the-crop-biology-breeding-and-applications-of-cannabis-sativa

\section{Hosted file}

Schilling2020_Table.pdf available at https://authorea.com/users/360584/articles/483690-thecream-of-the-crop-biology-breeding-and-applications-of-cannabis-sativa 


\section{Supplementary Material}

Supplementary Table 1. Chromosome lengths for each Cannabis genome assembly.

Supplementary Table 2. Available Cannabis sequencing data from the NCBI sequence read archive.

Supplementary Table 3. Available Cannabis organellar genome assemblies.

Hosted file

Schilling2020_SupplTables .pdf available at https://authorea.com/users/360584/articles/483690the-cream-of-the-crop-biology-breeding-and-applications-of-cannabis-sativa 